\title{
The Case of Avoidance to Accept the Inheritance in the Existence of the Heir Creditor
}

\author{
Khatia Gelashvili \\ PhD candidate in Law at Caucasus \\ International University
}

ARTICLE INFO

Article History:

Received 21.10.2021

Accepted 3.11.2021

Published 20.12.2021

Keywords:

Refusal to accept the

inheritance,

Creditor of the heir, Fulfillment of the obligation by the heir

\section{ABSTRACT}

The inheritance right is one of the basic human rights, which is protected and guaranteed by the Constitution. This fact implies that the state has certain obligations to recognize this right.

The article deals with the case where the heir does not want to receive the inheritance because the testator has creditors who seek to meet the obligations left by the testator. This issue has become more frequent in recent years, as it is not yet regulated at the legislative level, so I will offer recommendations to the public. The study reveals the difficulties associated with this case of inadmissibility of the estate, when the main purpose of the heir is to avoid liability to the creditors of the testator, both by will and by law. The study discusses in each case, the inadmissibility of the entire estate, as well as the inadmissibility of part of it, as well as the case of liability to several creditors of the heir. I think the discussion of this issue will be really new for the Georgian legislation, because the Civil Code does not fully regulate this topic and it can be boldly said that the research issue will not lose its relevance in practice. 


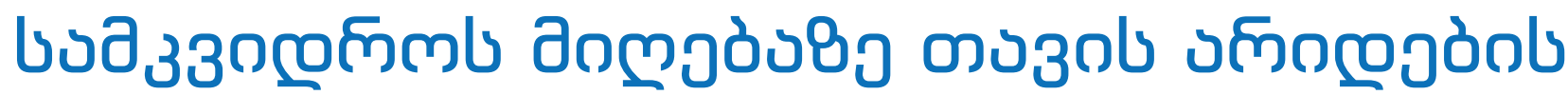

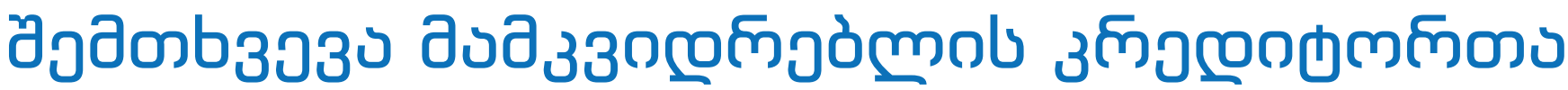

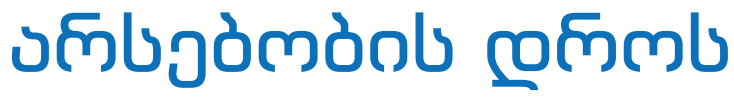

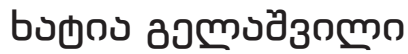

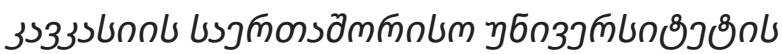

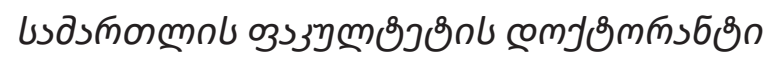

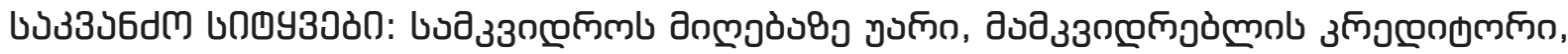
ة

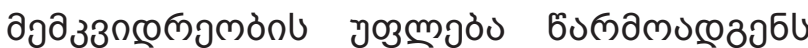

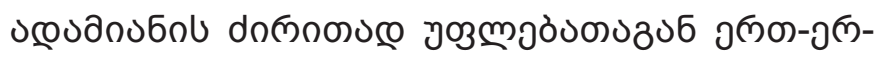

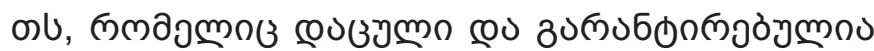

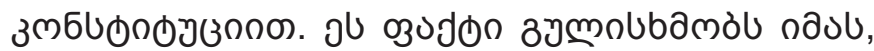

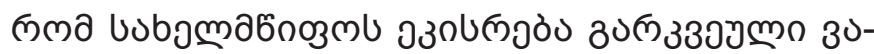

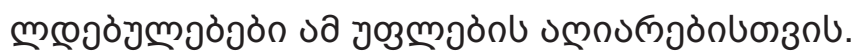

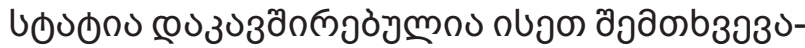

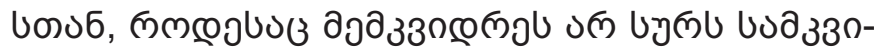

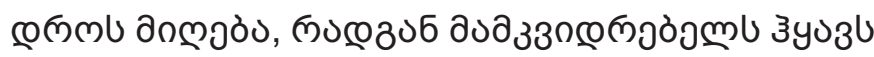

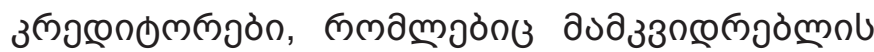

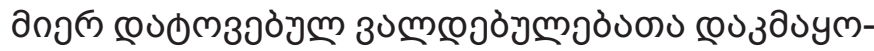

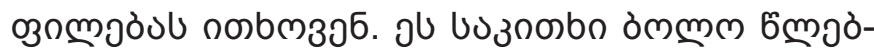

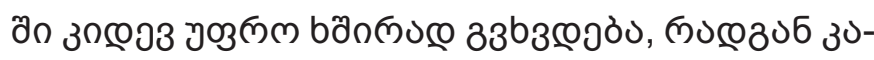

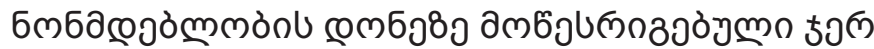

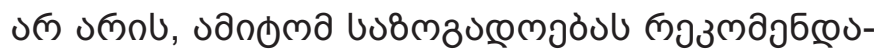

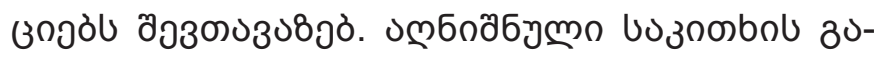

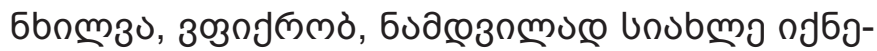

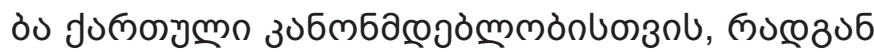

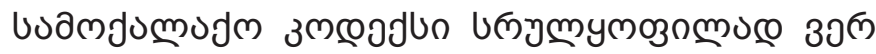

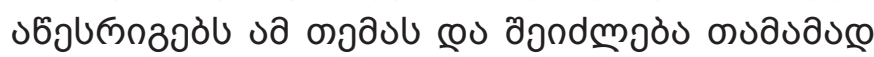

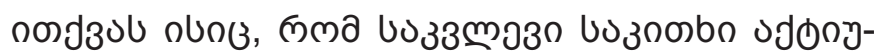

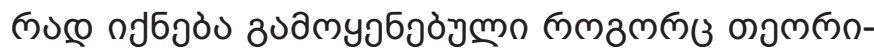

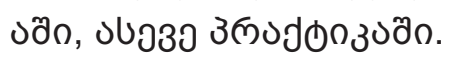

$$
* * *
$$

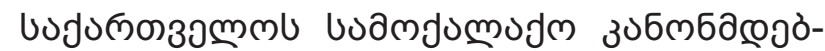

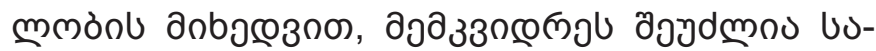

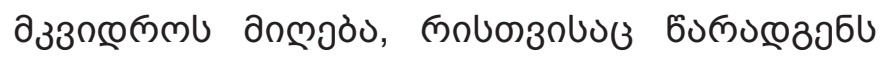

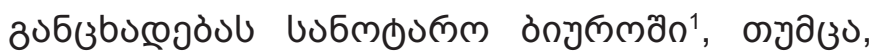

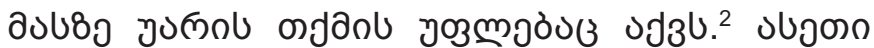

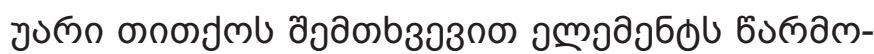

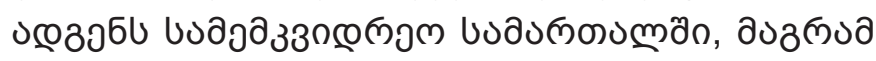

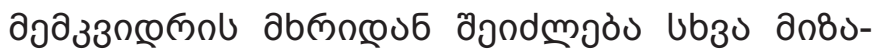

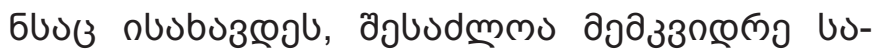

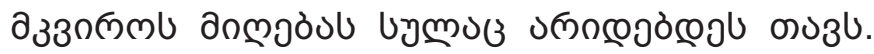

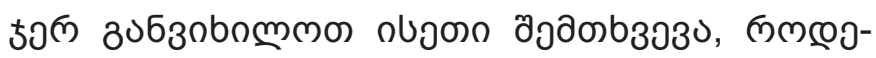

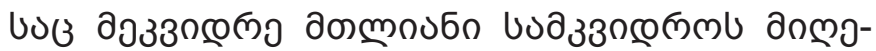

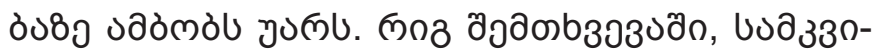

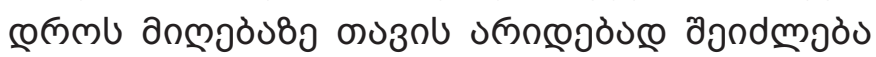

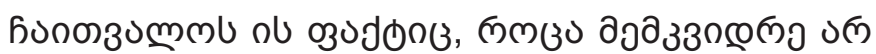

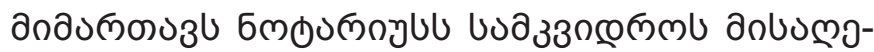

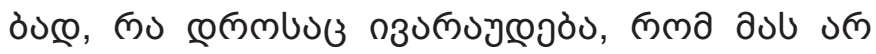

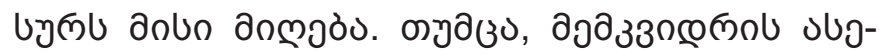

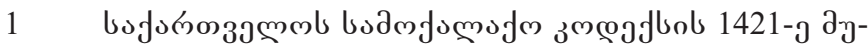
bmols a $\mathrm{g}^{-2} 3 \mathrm{y}^{6} \mathrm{j}^{\mathrm{o}}$.

2 у јб

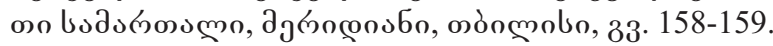




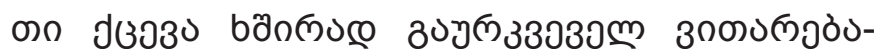

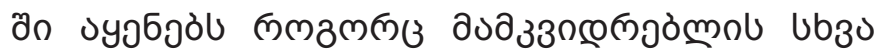

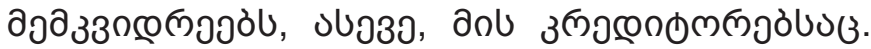

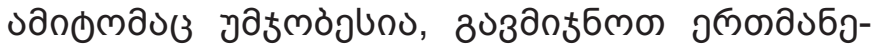

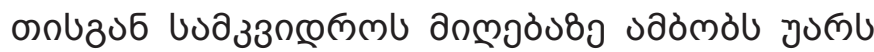

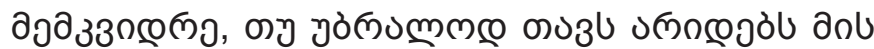

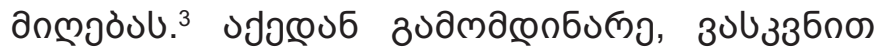

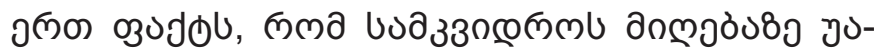

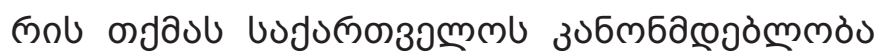

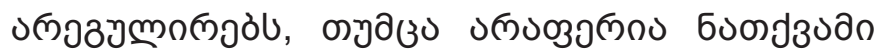

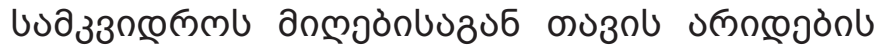

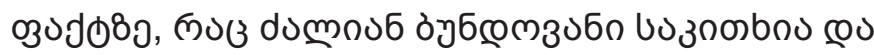

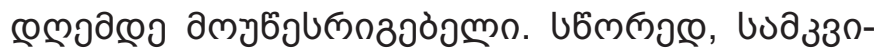

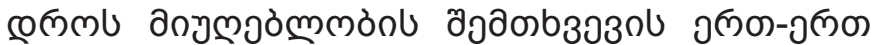

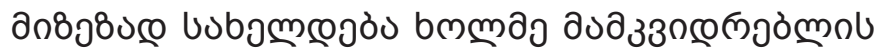

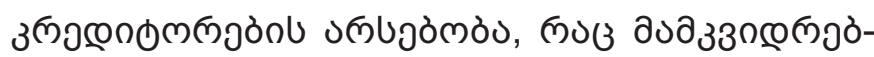

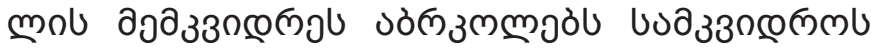

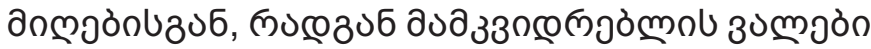

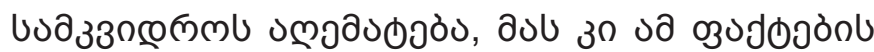

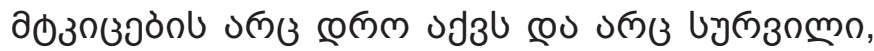

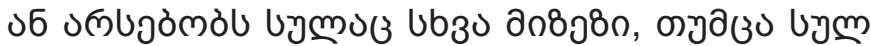

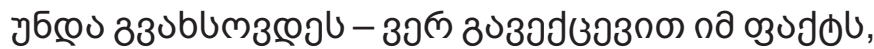

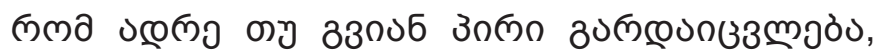

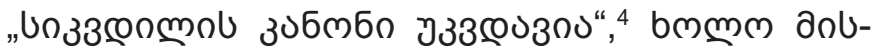

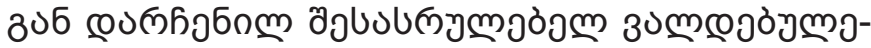

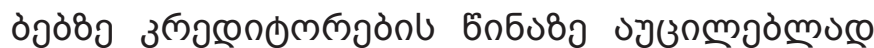

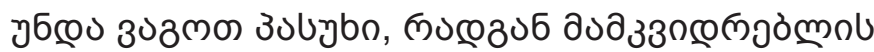

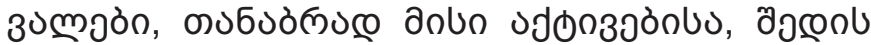

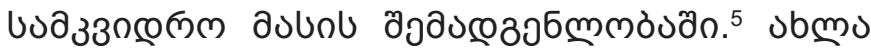

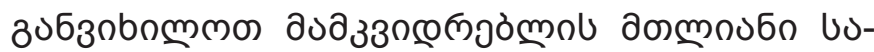

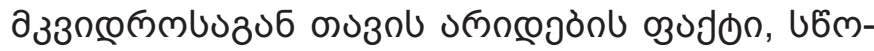

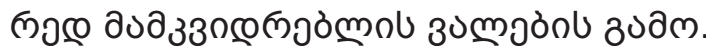

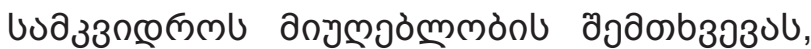

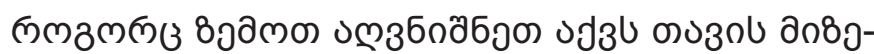

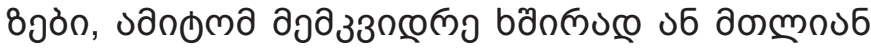

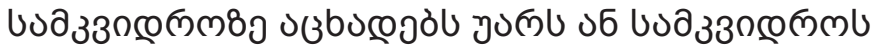

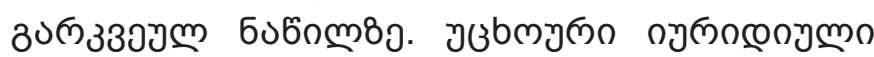

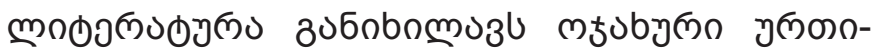

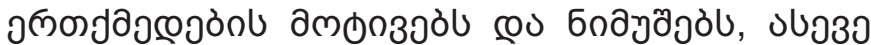

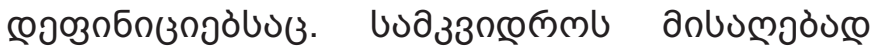

3

od $39,33.158$.

Adam J. Hirsch, American History of Inheritance Law, OXFORD INTERNATIONAL ENCYCLOPEDIA OF LEGAL HISTORY, Stanley N. Katz ed., 2009, 235. https:// papers.ssrn.com/sol3/papers.cfm?abstract $\mathrm{id}=982428$ [22.08.2020].

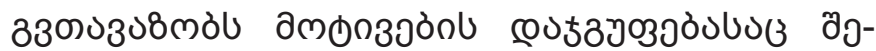

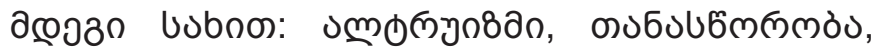

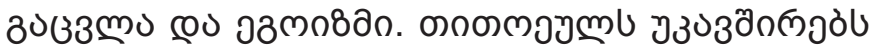

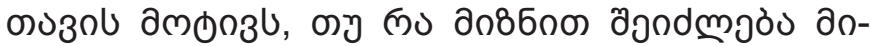

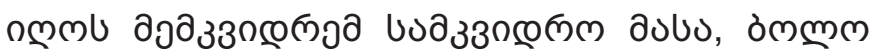

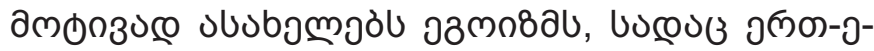

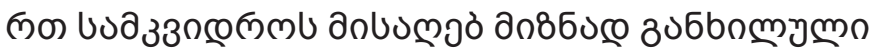
১ปзు n

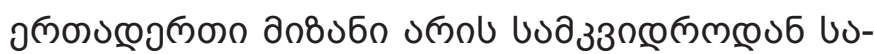

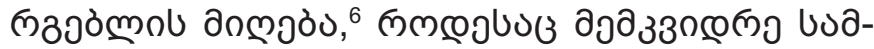

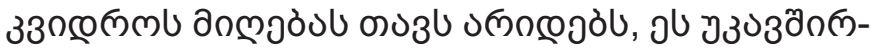

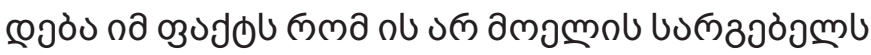

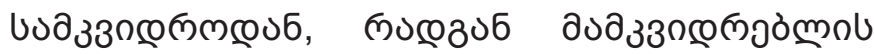

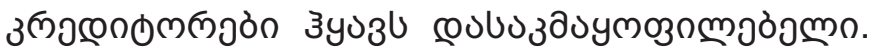

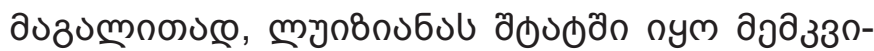

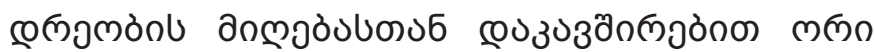

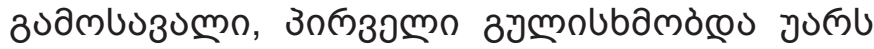

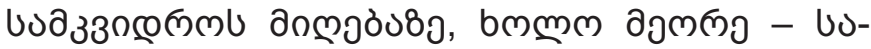

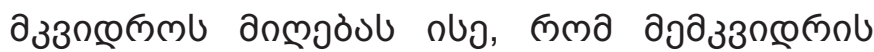

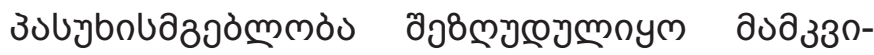

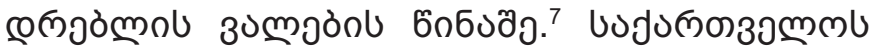

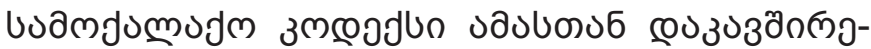

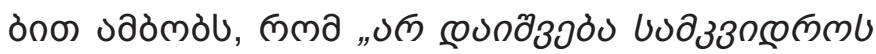

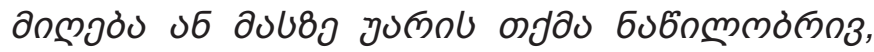

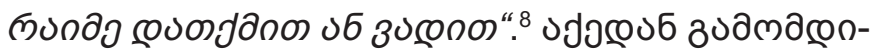

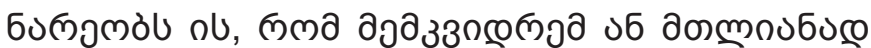

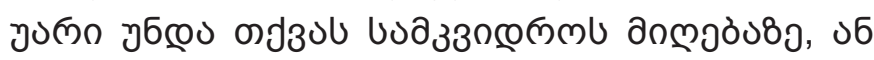

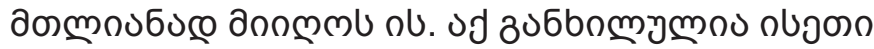

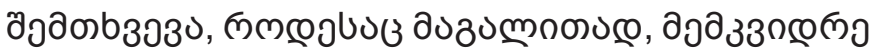

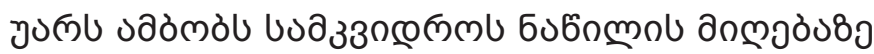

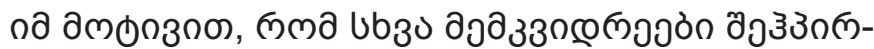

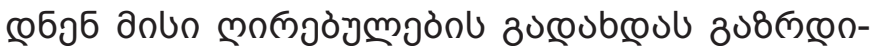

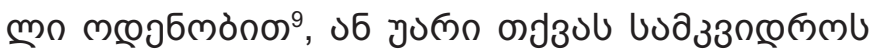

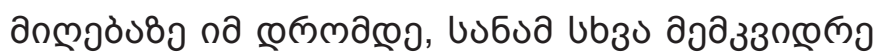

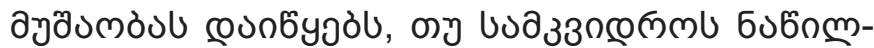

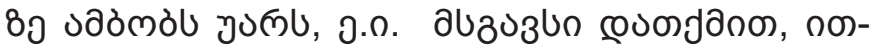

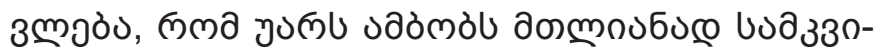

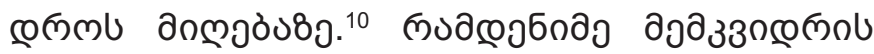

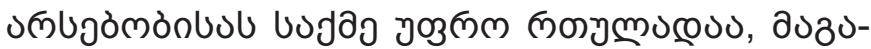

6 Sousa, L. The family inheritance process: motivations and patterns of interaction, 2010. https://www.researchgate. net/publication/226734632 The family inheritance process Motivations and patterns of interaction [11.06.2020].

7 Cavanaugh K.W., Problems In The Law of Succession: Creditors' Rights, 48 La. L. Review, 1988, 1100-1101.

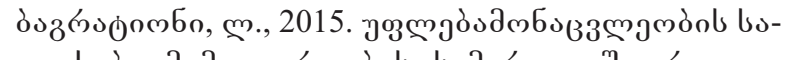

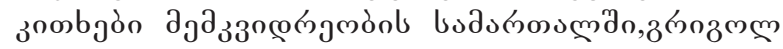

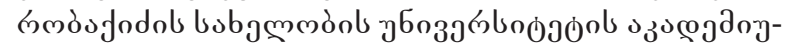

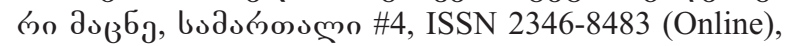
oòngobo, 101.

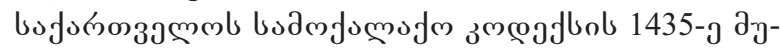
bmo.

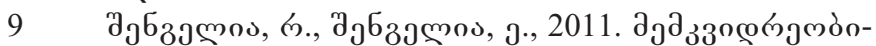

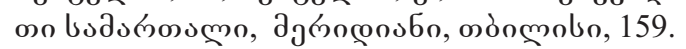

10 of39, $33.159-160$. 


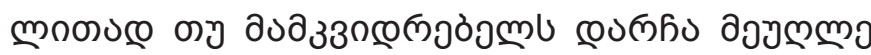

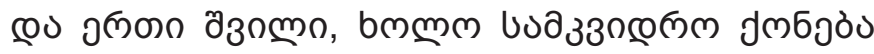

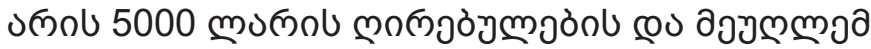

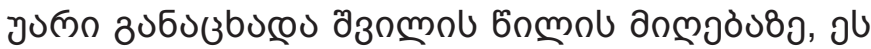

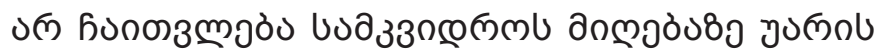

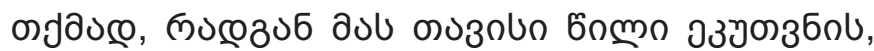

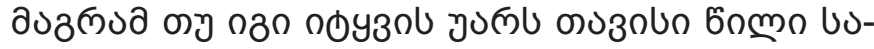

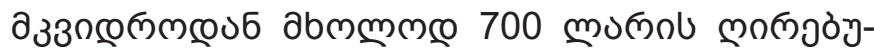

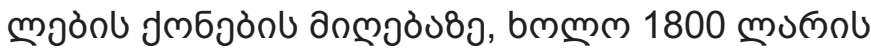

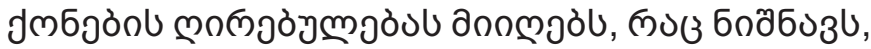

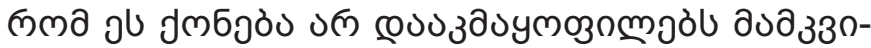

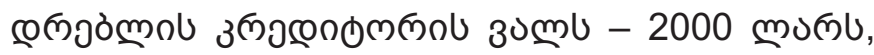

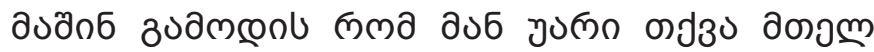

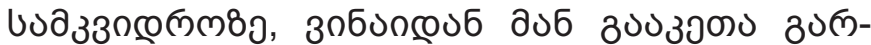

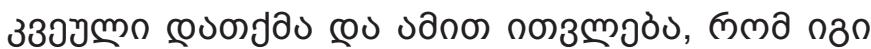

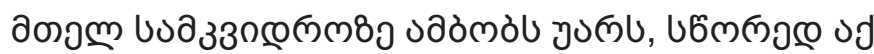

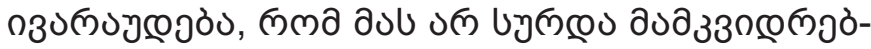

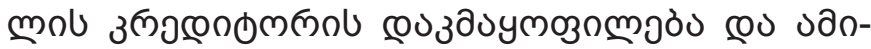

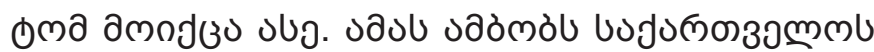

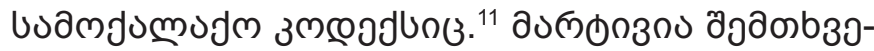

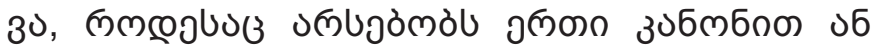

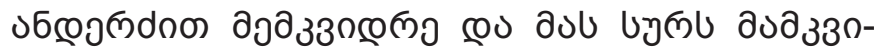

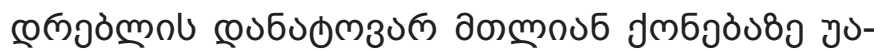

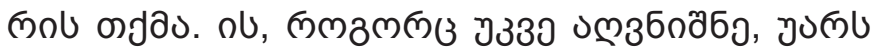

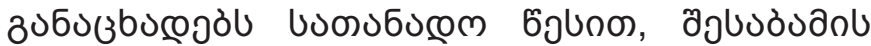

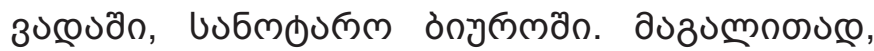

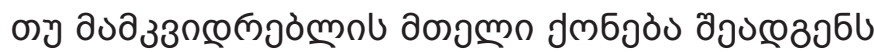

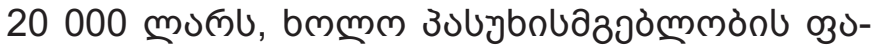

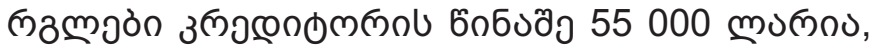

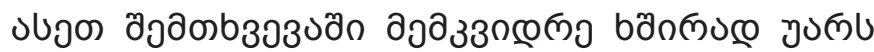

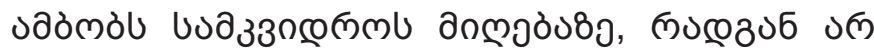

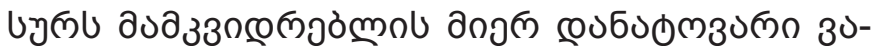

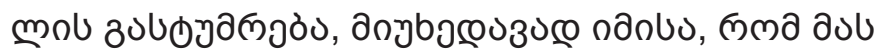

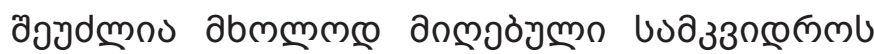

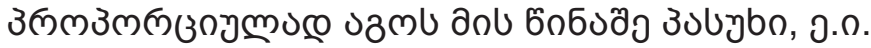

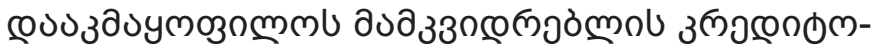

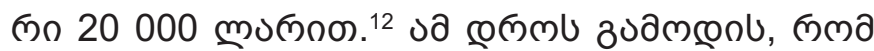

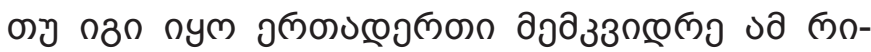

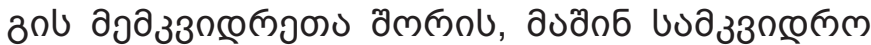

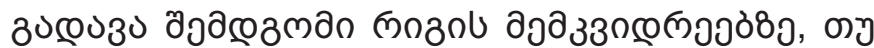

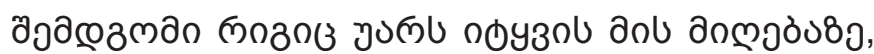

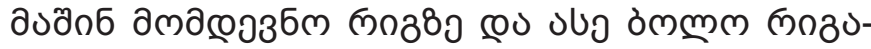

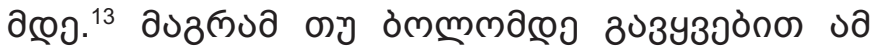

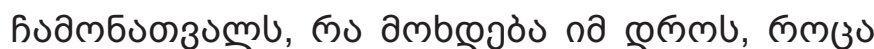

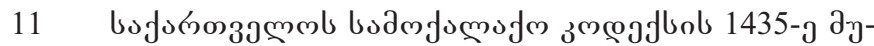
bemo.

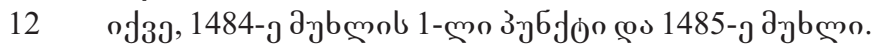

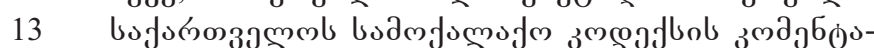

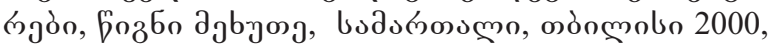
485.

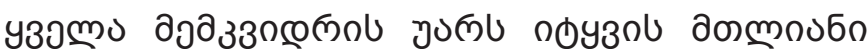

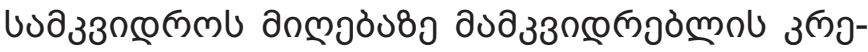

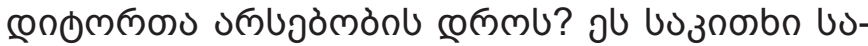

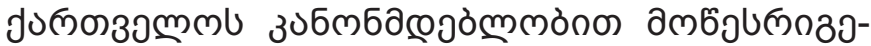

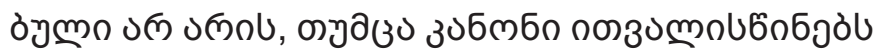
০

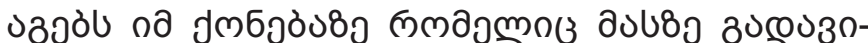

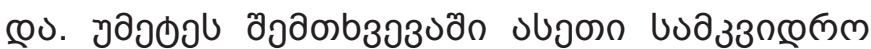

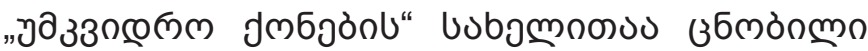

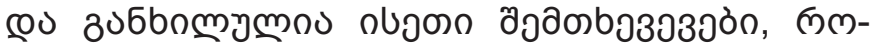

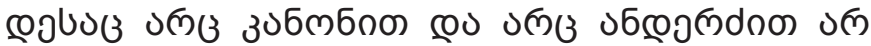

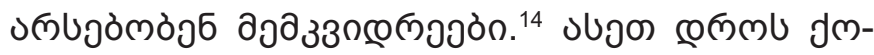

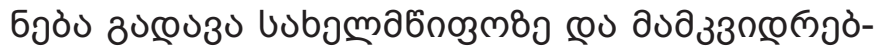

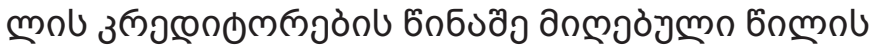

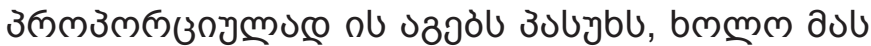

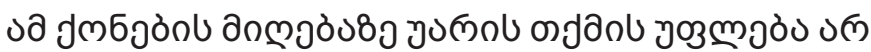

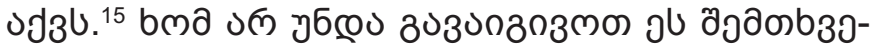

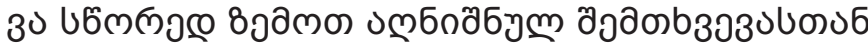

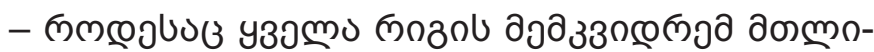

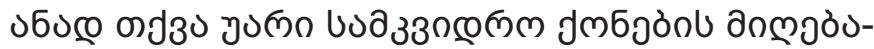

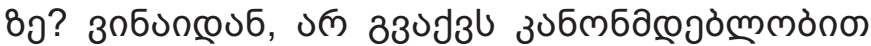

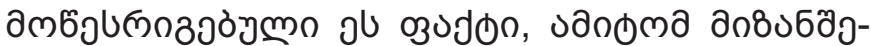

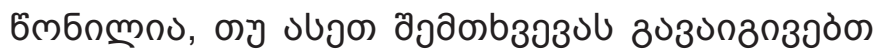

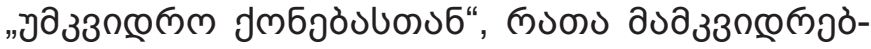

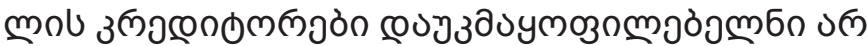

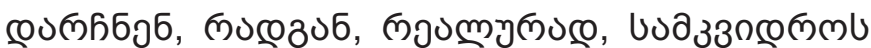

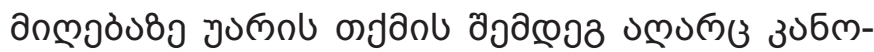

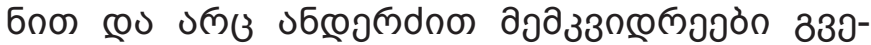

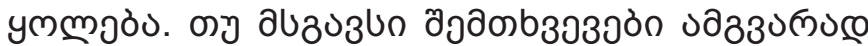

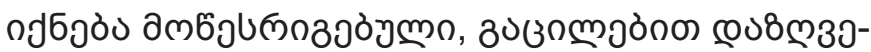

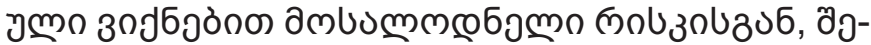

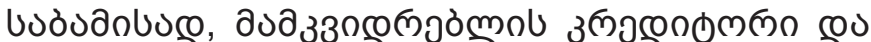

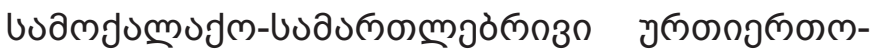

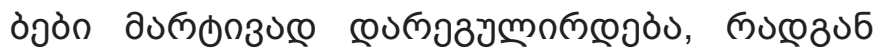

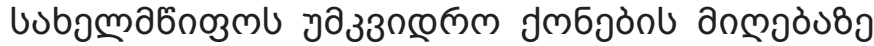

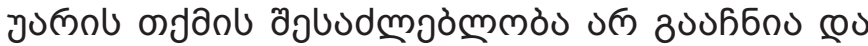

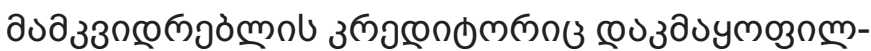

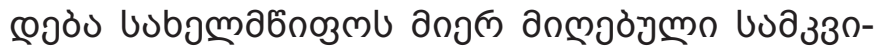

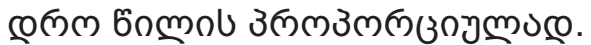

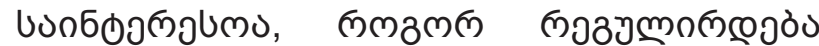

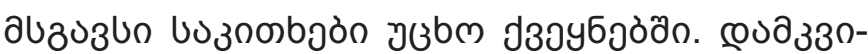

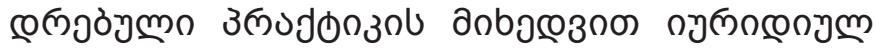

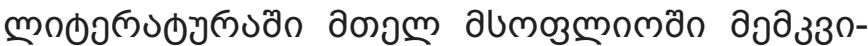

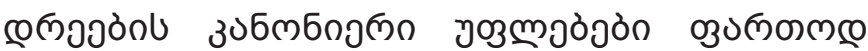

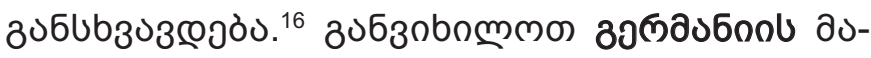

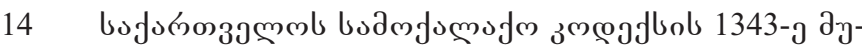
bemo.

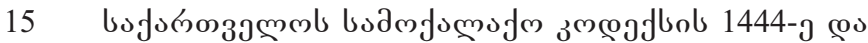
1492-g dybmo.

16 Ellul, A., Pagano, M., Panunzi, F., Inheritance Law and
} 


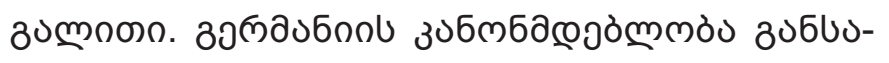

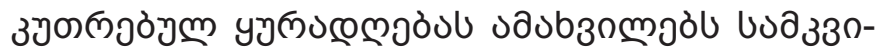

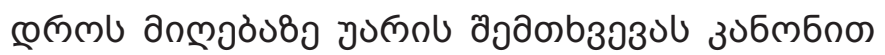

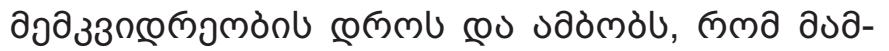

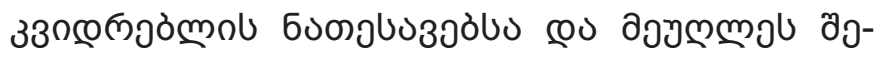

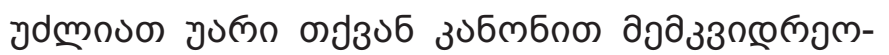

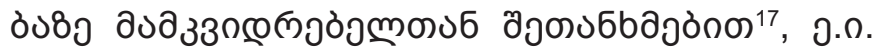

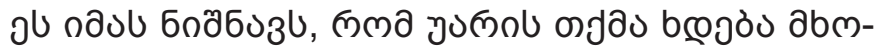

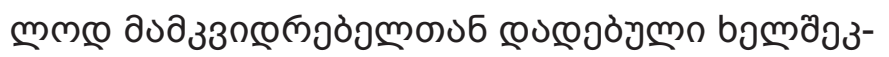

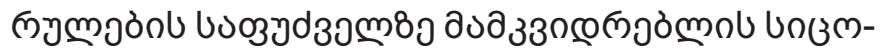

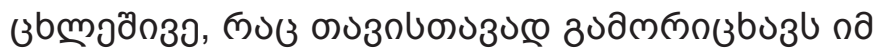

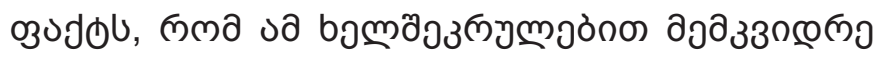

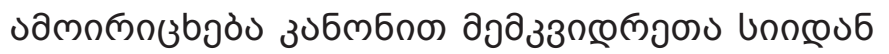

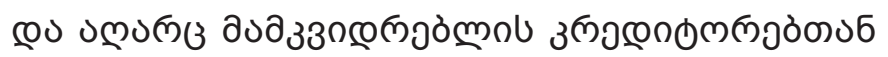

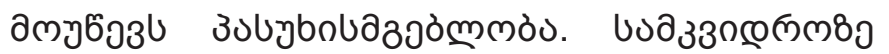

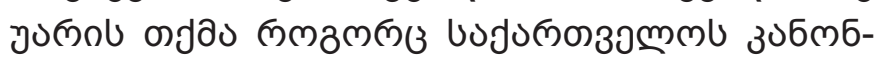

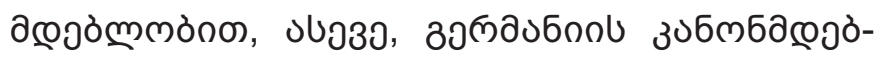

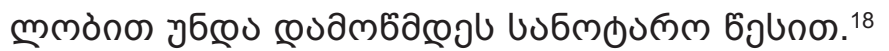

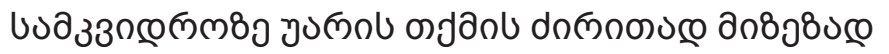

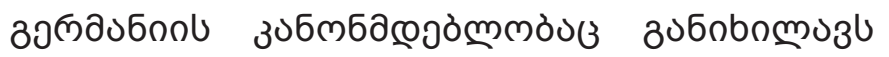

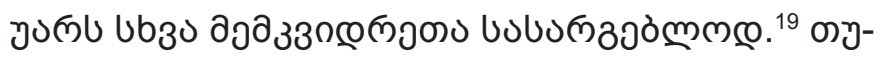

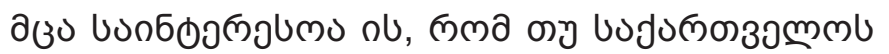

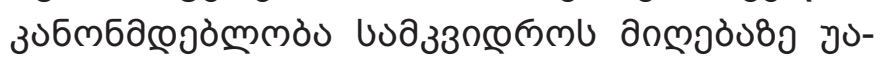

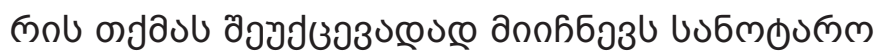

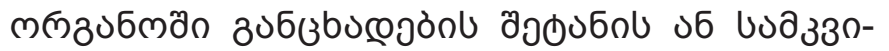

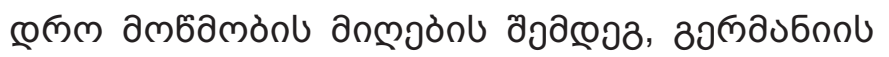

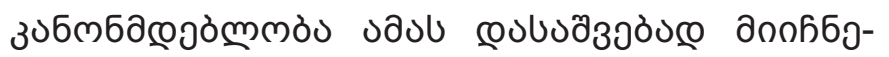

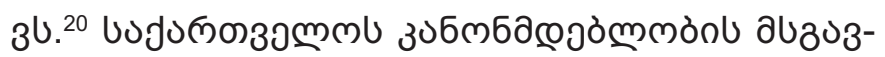

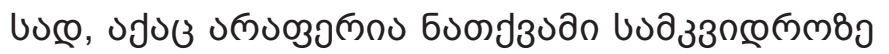

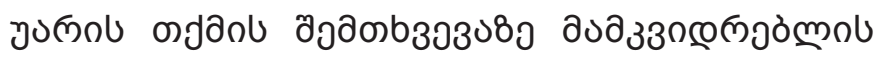

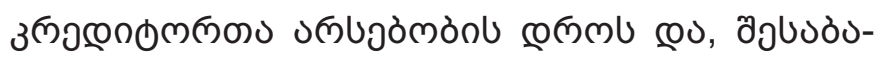

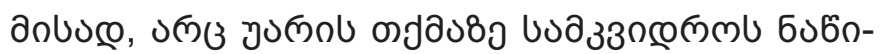

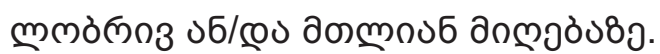

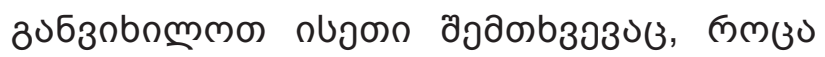

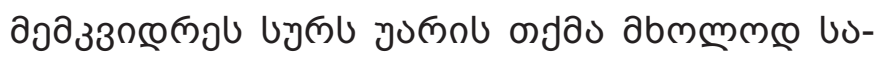

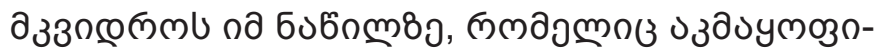

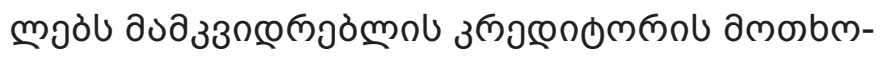

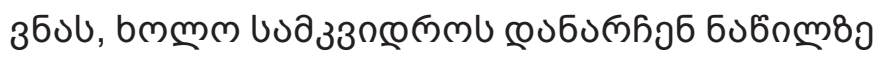

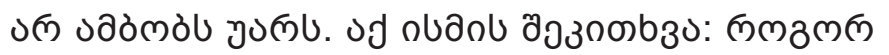

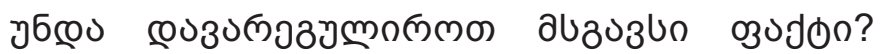

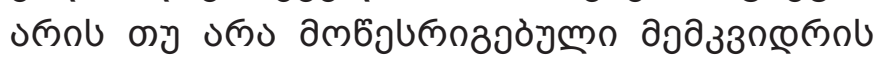

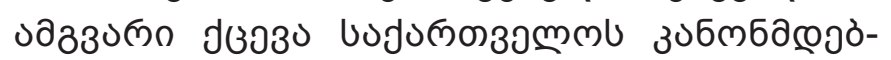

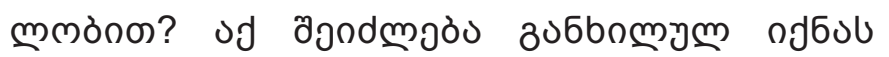

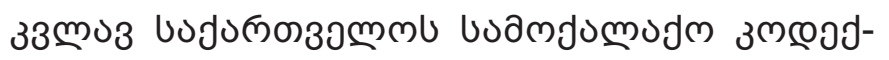

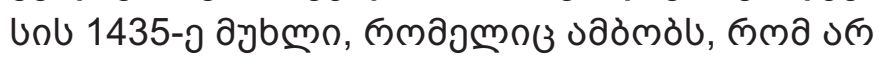

Investment in Family Firms, 2010.

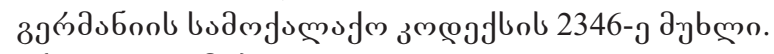
of39, 2348-э วybmo.

of39, 2350-э dybmo.

of39, 2351-э วybmo.

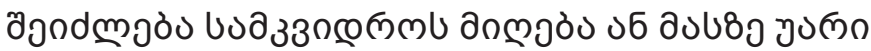

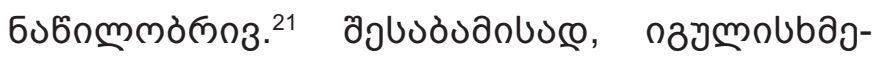

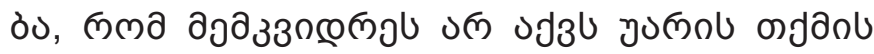

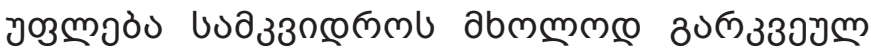

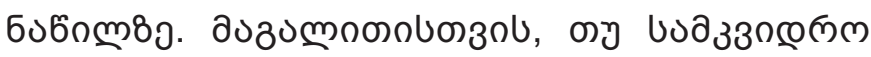

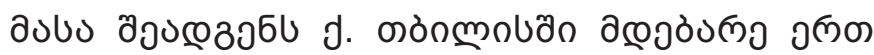

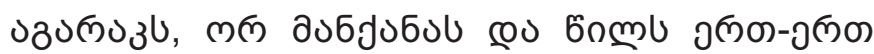

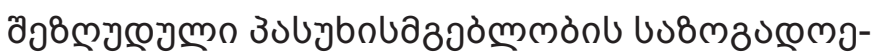

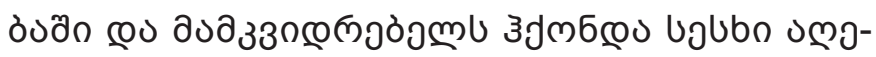

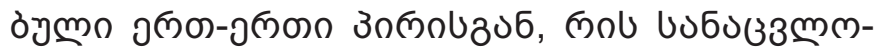

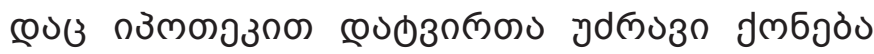

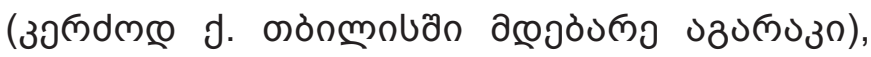

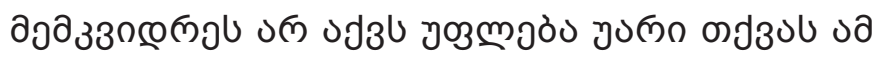

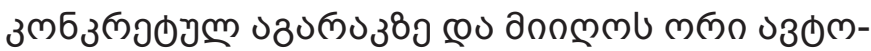

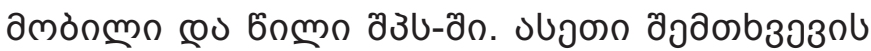

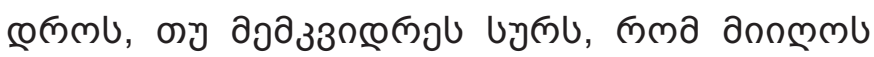

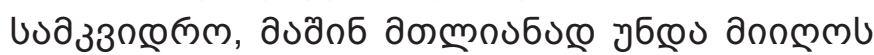

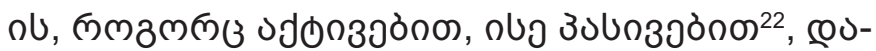

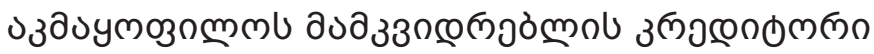

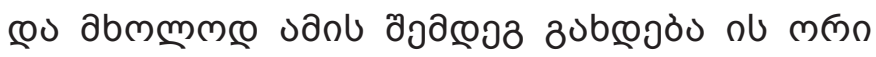

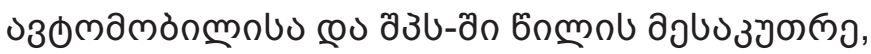

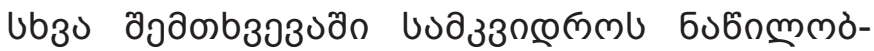

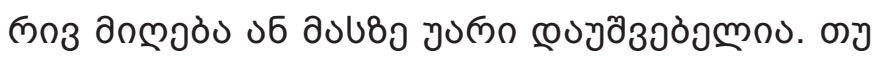

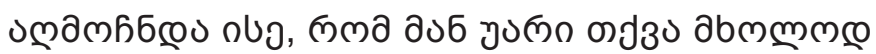

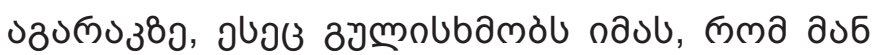

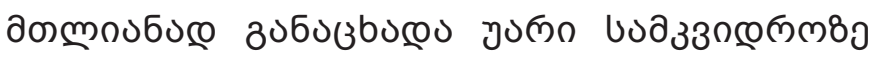

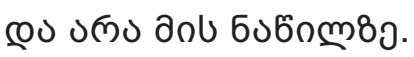

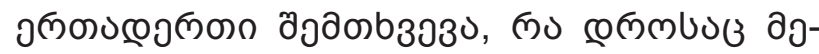

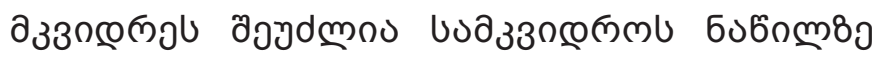

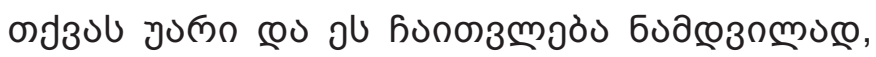

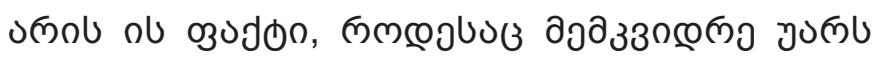

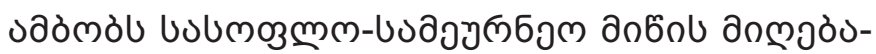

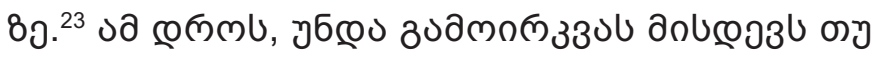

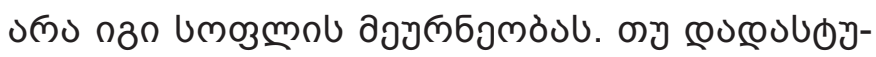

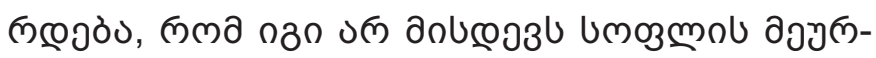

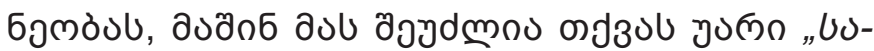

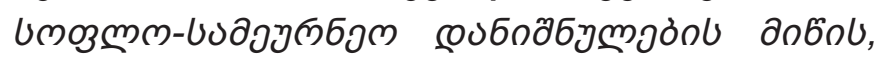

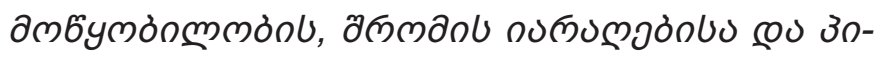

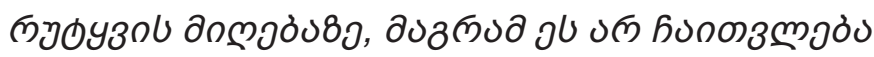

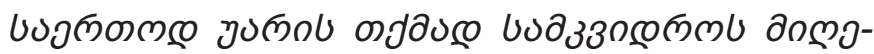
oง80".24

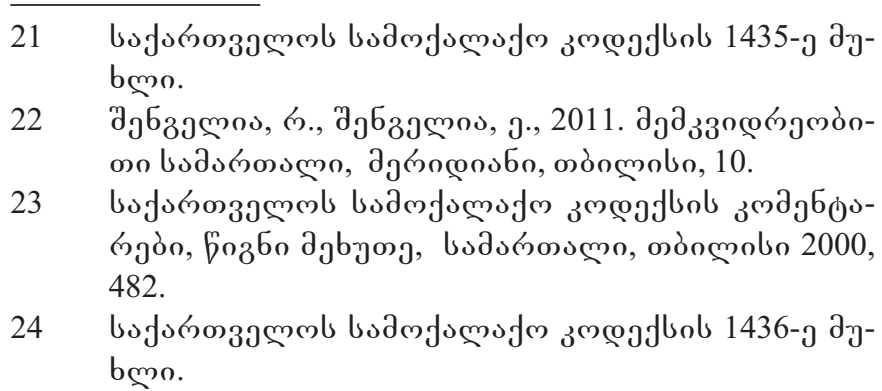




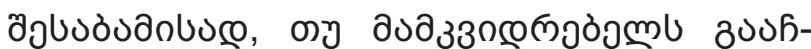

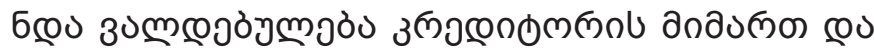

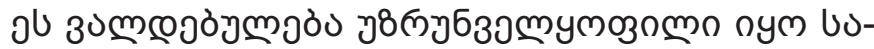

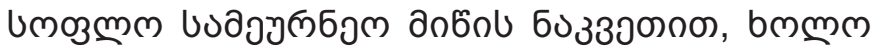

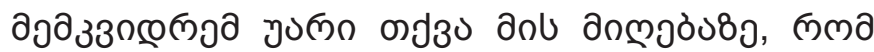

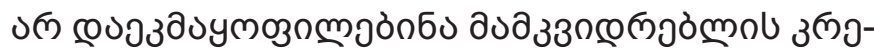

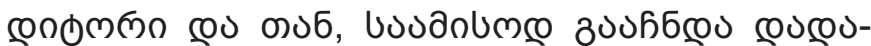

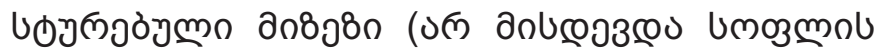

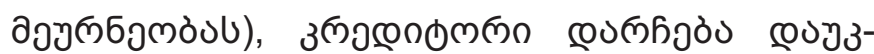

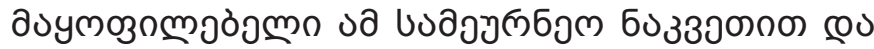

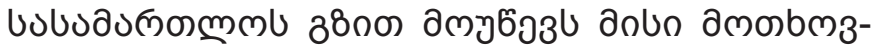

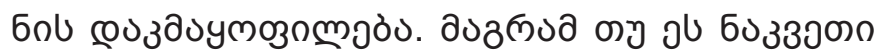

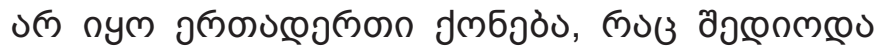

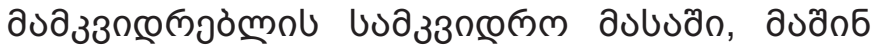

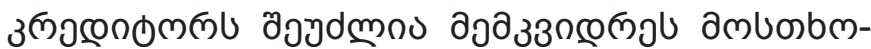

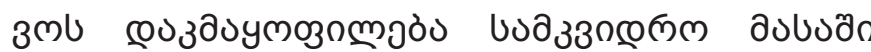

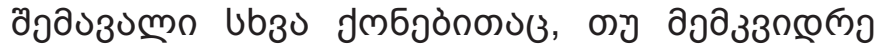

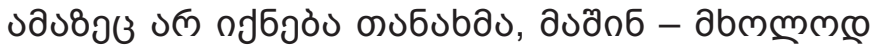

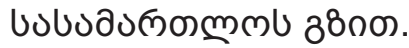

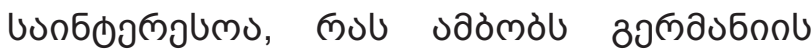

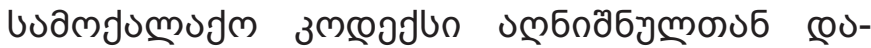

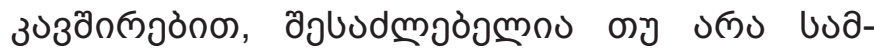

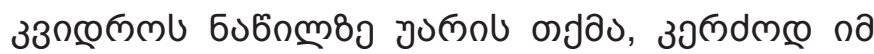

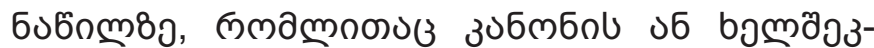

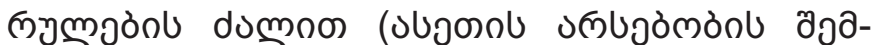

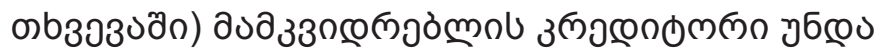

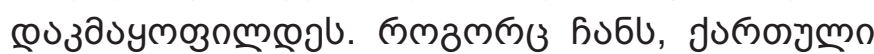

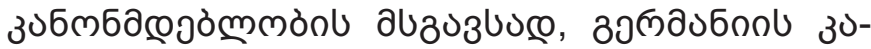

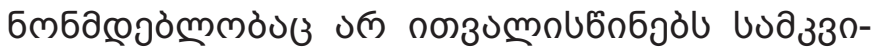

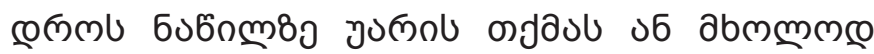

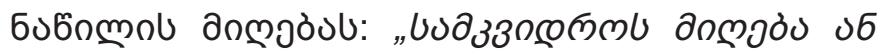

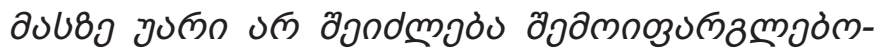

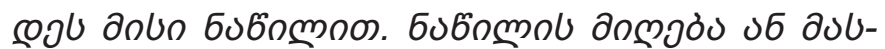

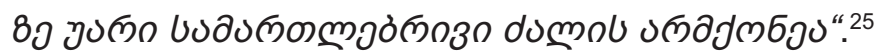

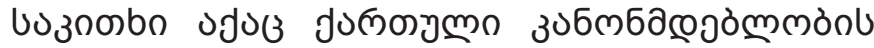

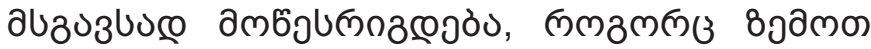

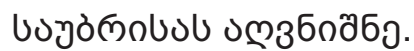

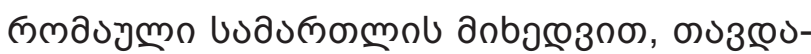

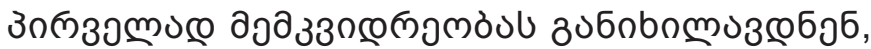

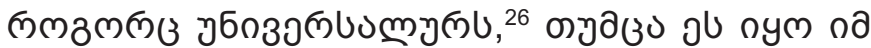

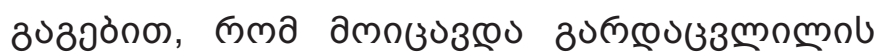

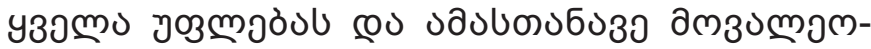

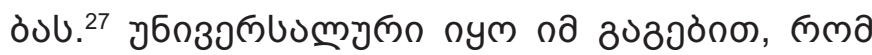

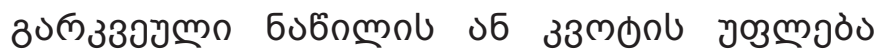

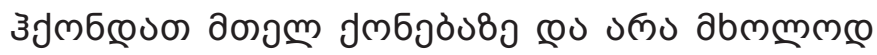

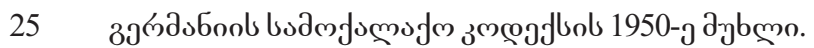

26 Domingo, R., The Roman Law of Succession. An Overview, University of Navarra - School of Law, 2017, $2-3$. of 39,33 2-3.

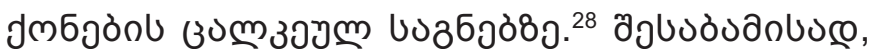

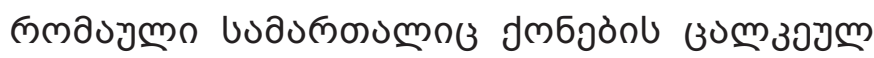

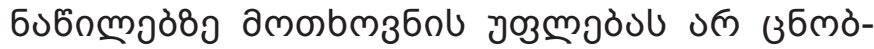

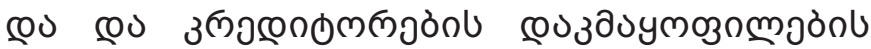

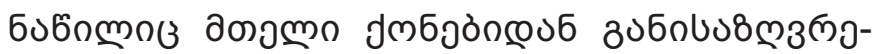

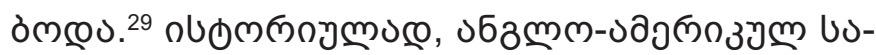

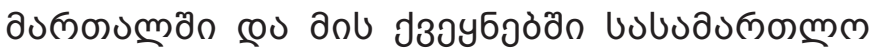

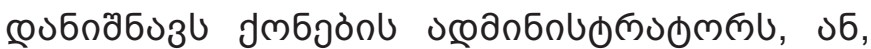

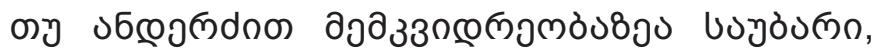

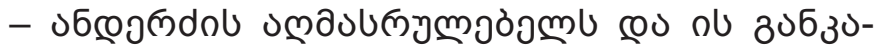

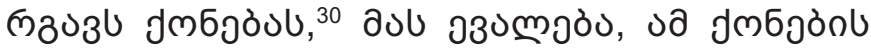

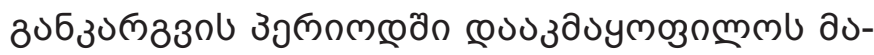

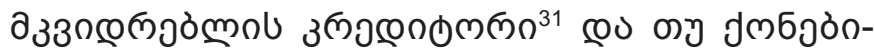

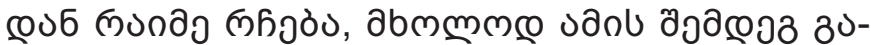

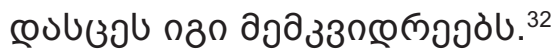

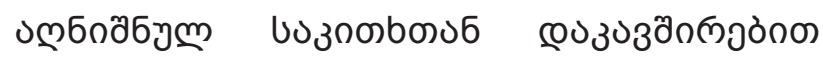

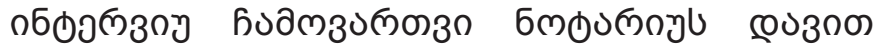

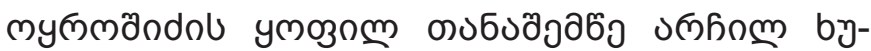

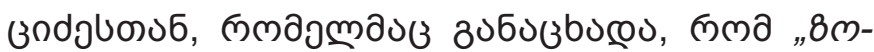

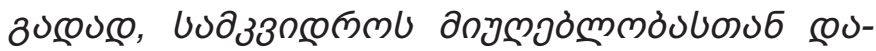

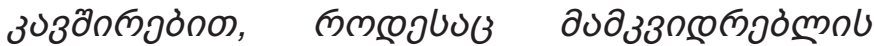

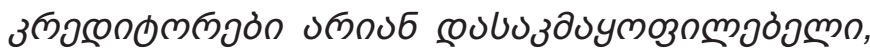

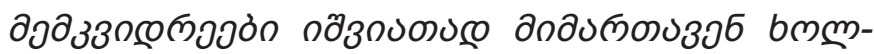

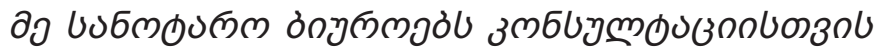

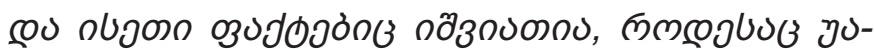

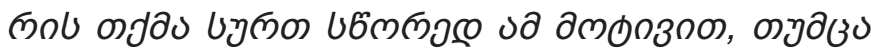

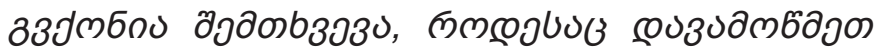

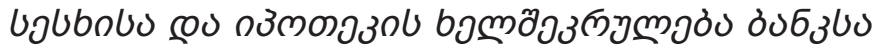

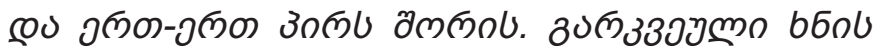

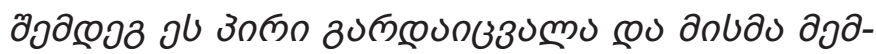

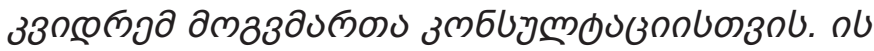

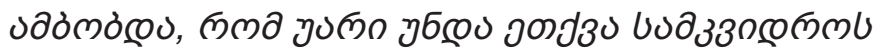

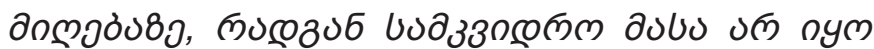

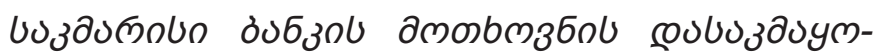

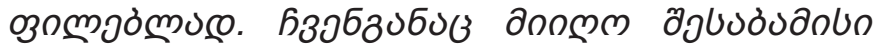

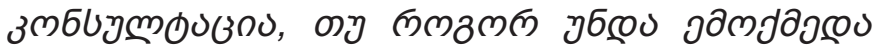

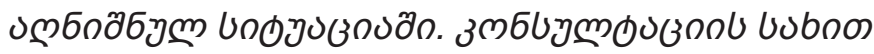

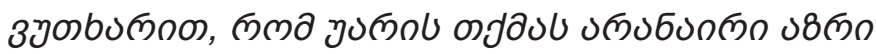

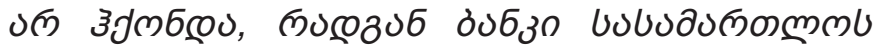

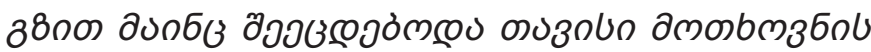

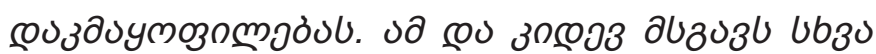

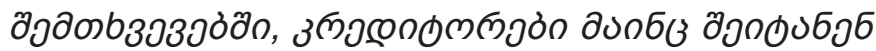

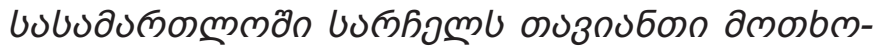

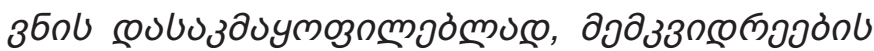

\begin{tabular}{|c|c|}
\hline 28 & oł39, oł3ง 33. 2 . \\
\hline 29 & of39, $33.2-3$ \\
\hline 30 & $\begin{array}{l}\text { Wormser R. A., Burchard A., ADMINISTRATION OF } \\
\text { GERMAN DECENTENTS' ESTATES, 1934, 40-41. }\end{array}$ \\
\hline 31 & of3ง. \\
\hline 3 & od39. \\
\hline
\end{tabular}




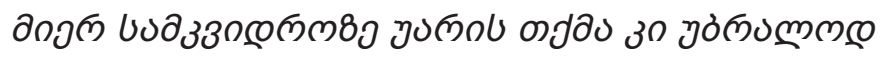

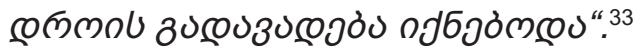

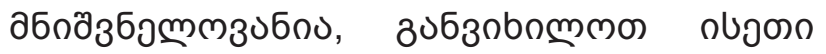

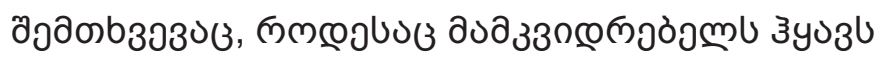

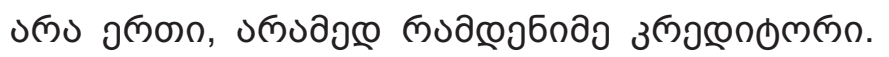

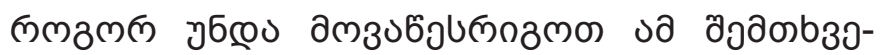

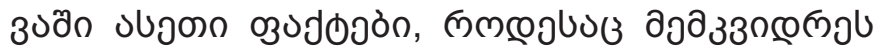

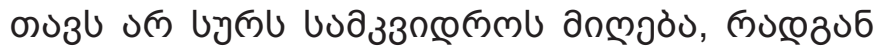

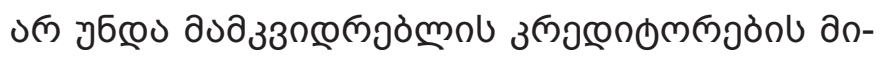

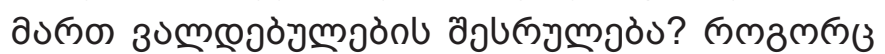

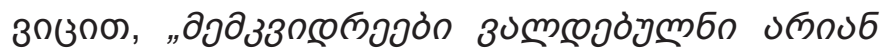

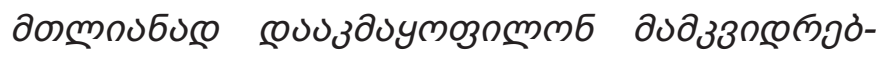

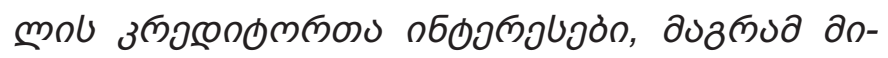

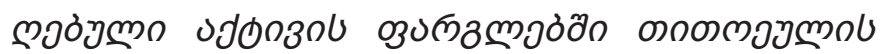
бомо зпмзмпзолмико",

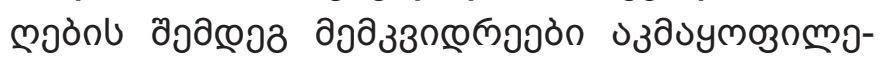

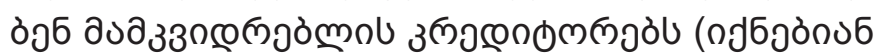

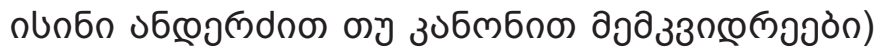

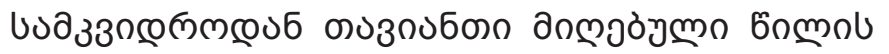

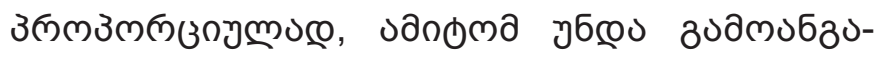

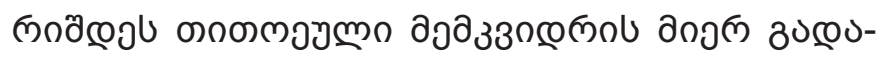

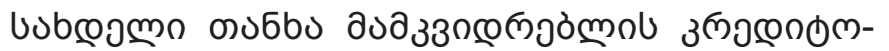

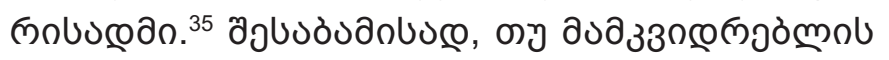

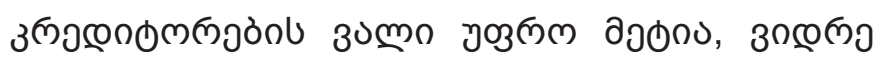

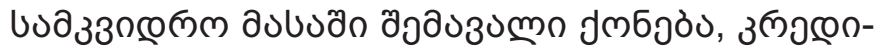

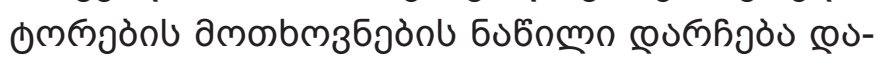

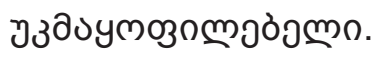

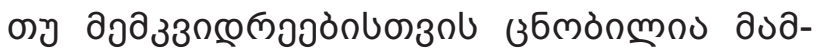

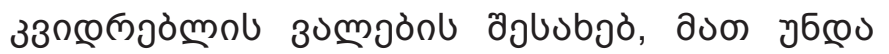

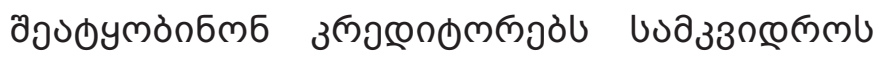

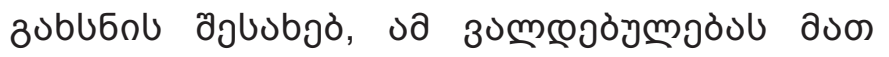

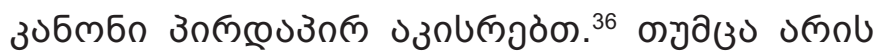

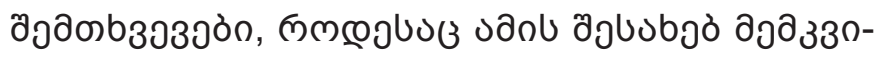

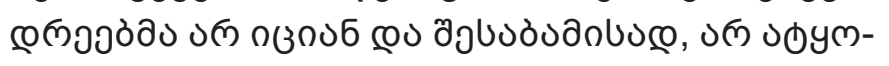

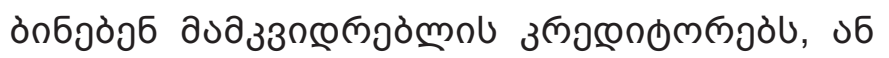

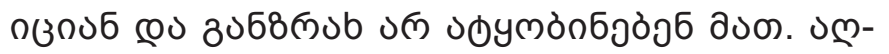

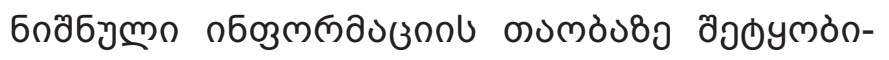

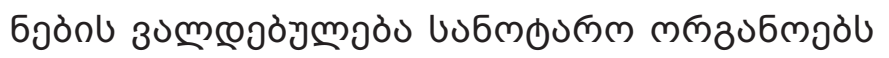

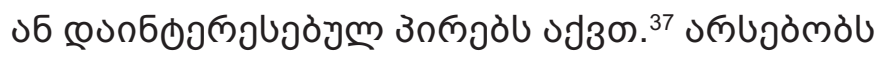

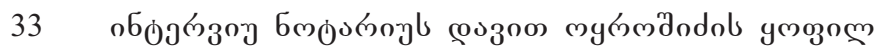

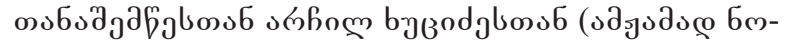

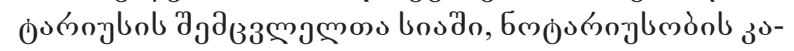

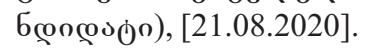

34 bofornom 39 mmb bodm bermo.

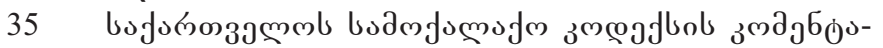

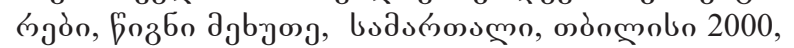
33. 514 .

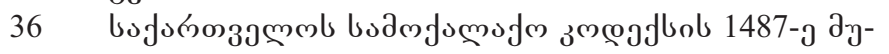
bemo.

37 д

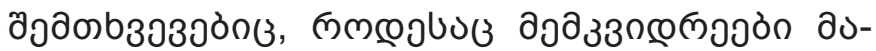

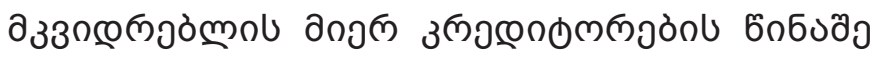

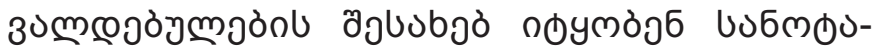

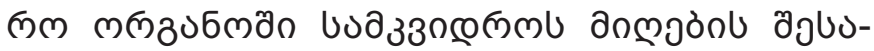

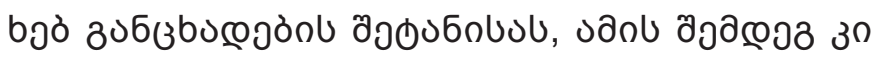

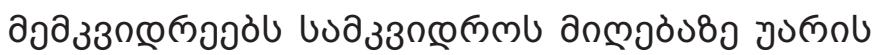

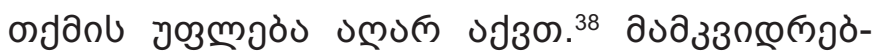

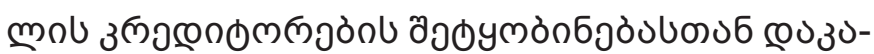

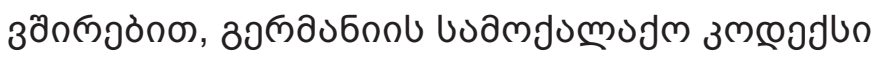

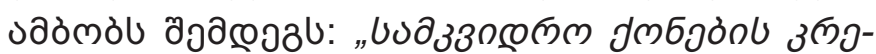

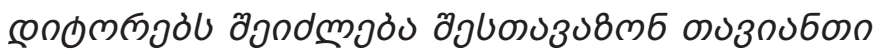

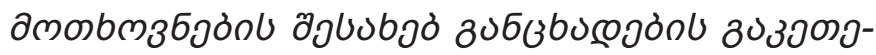

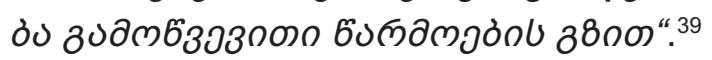

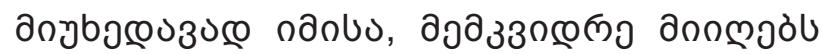

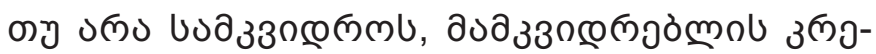

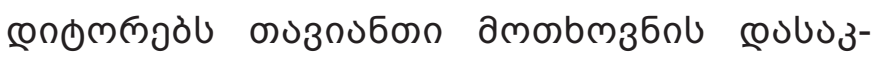

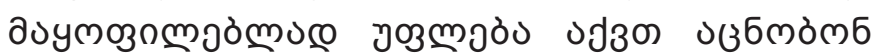

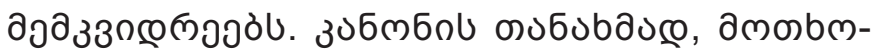

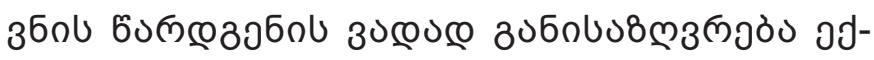

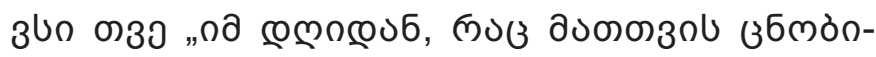

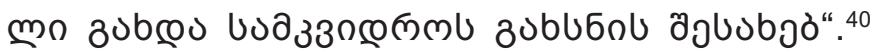

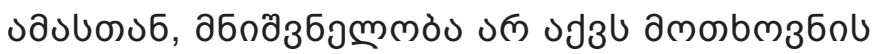

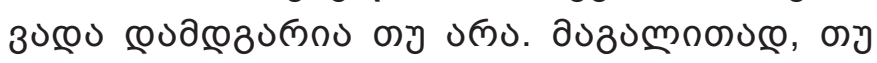

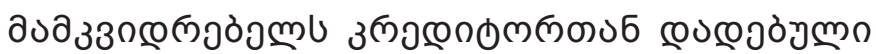

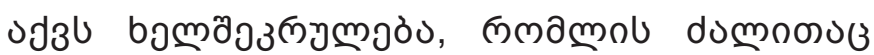

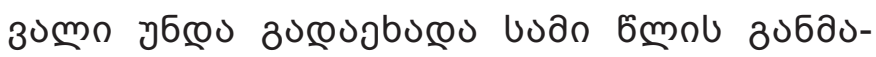

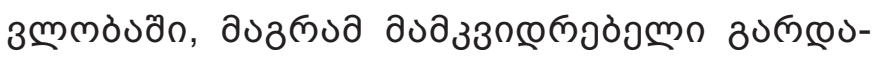

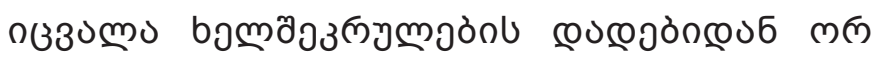

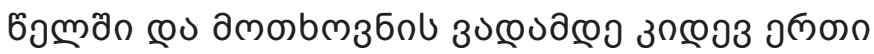

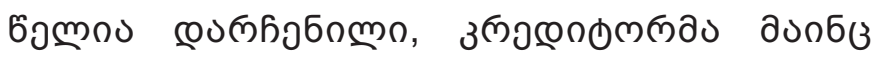

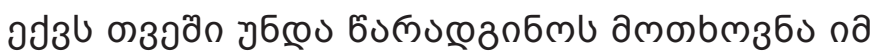

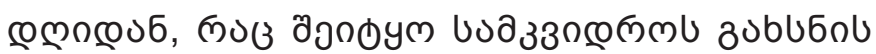
Әobubod. ${ }^{41}$

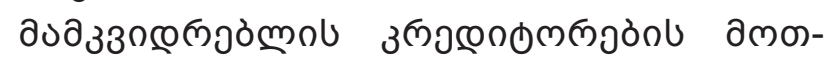

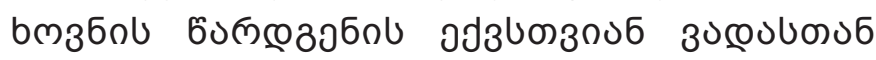

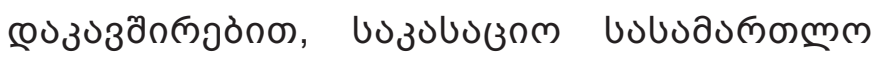

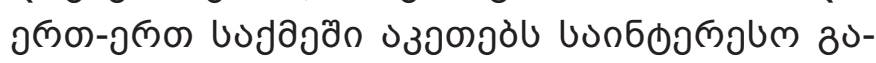

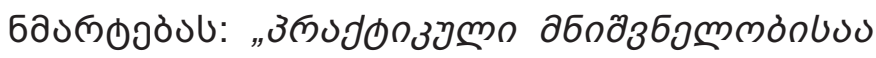

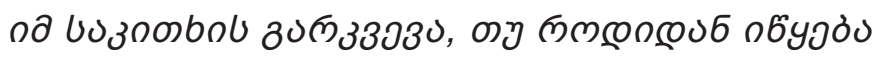
৬৬з-০ 1488.1 ау

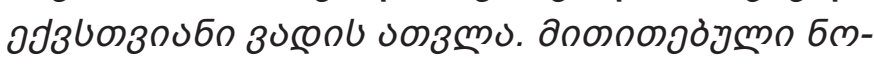

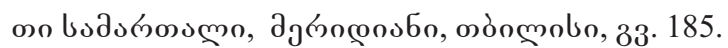

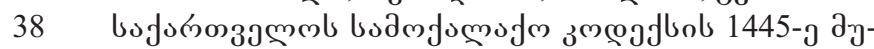
bmo.

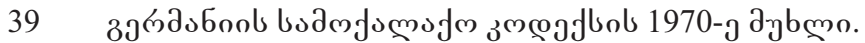

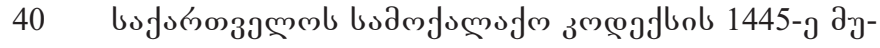
bemo.

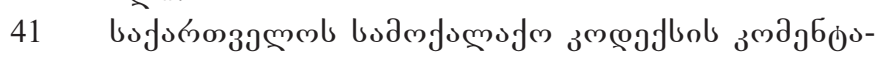

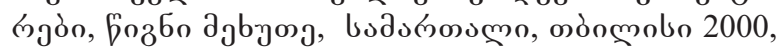
33. 516 . 


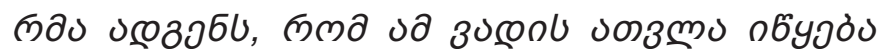

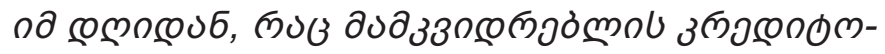

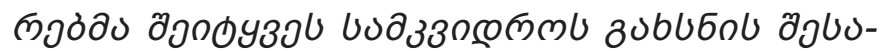

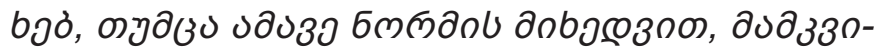

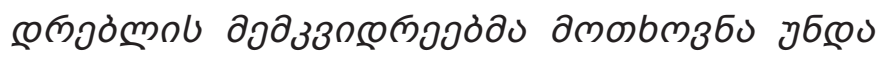

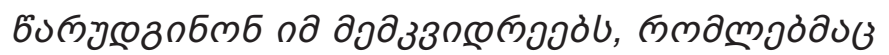

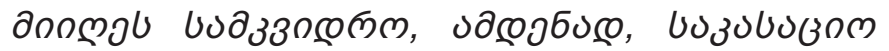

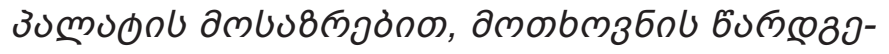

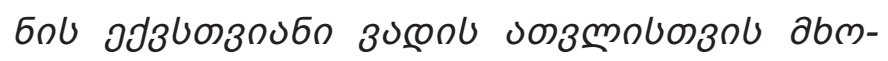

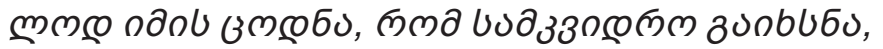

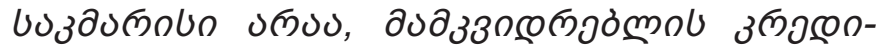

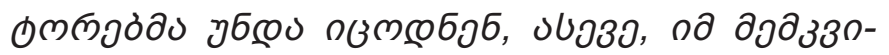

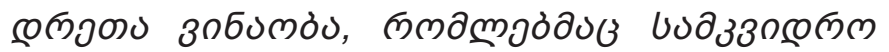

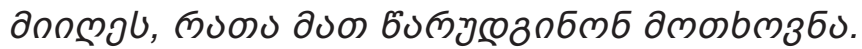

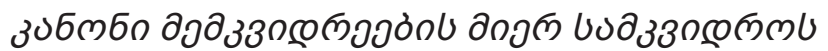

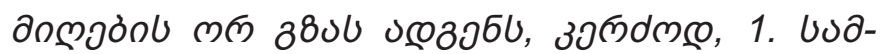

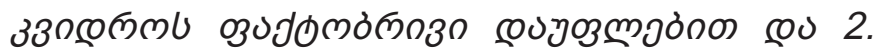

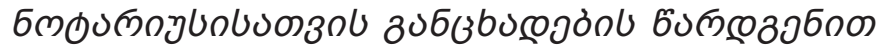

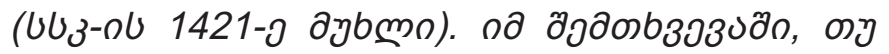

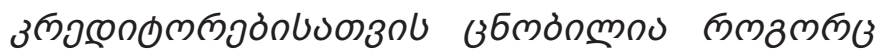

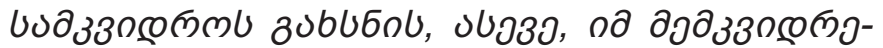

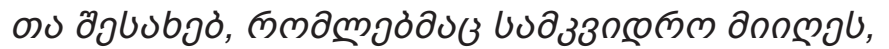

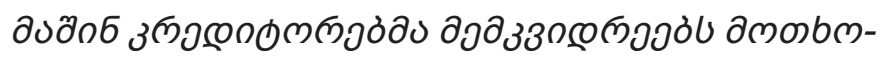

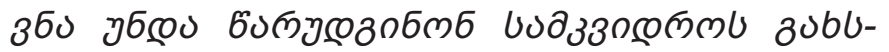

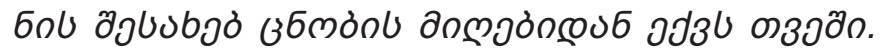

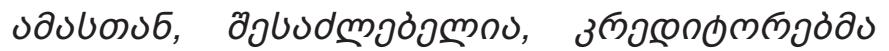

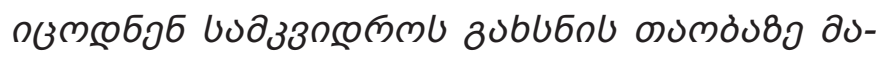

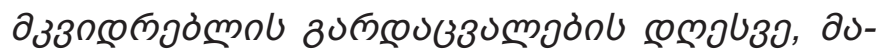

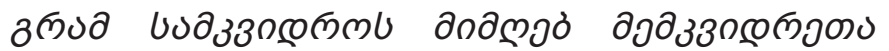

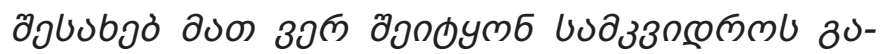

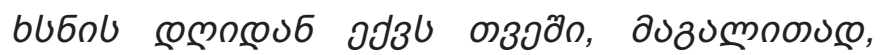

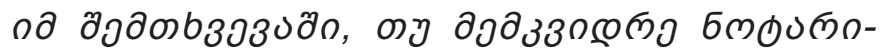

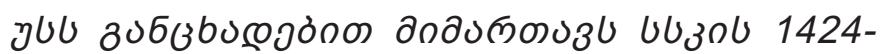

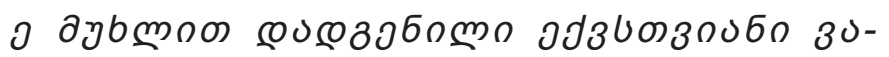

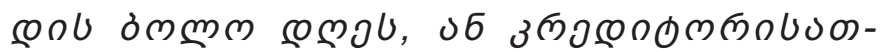

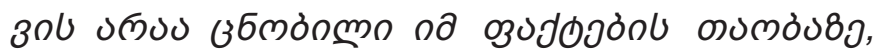

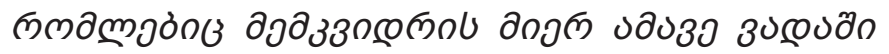

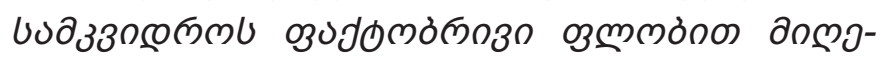

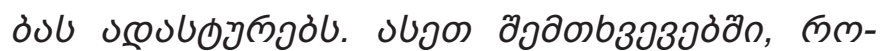

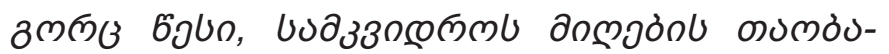

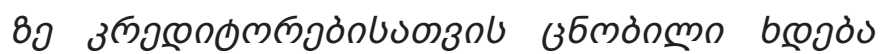

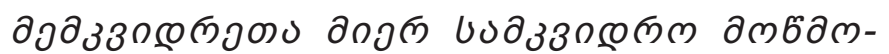

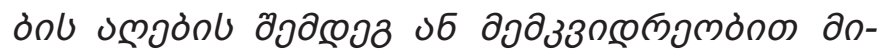

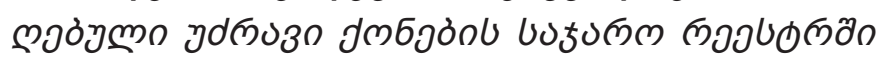

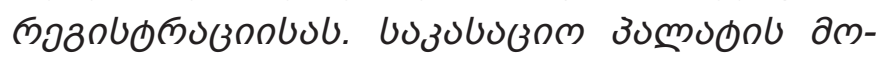

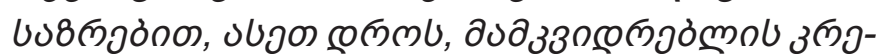

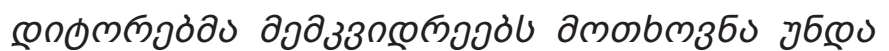

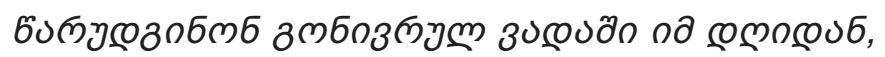

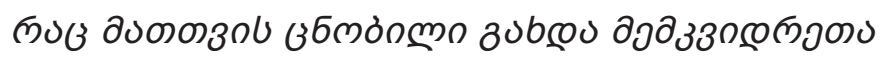

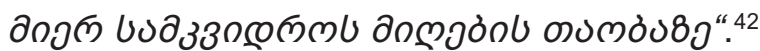

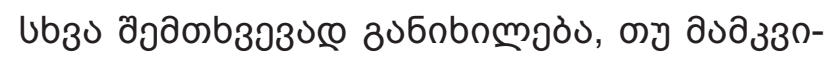

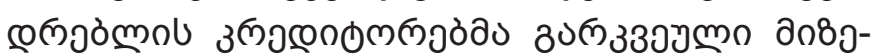

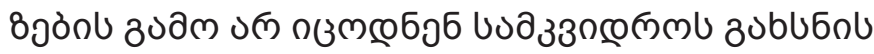
ajusbad cou aunajl ammbmzбnl cousozman

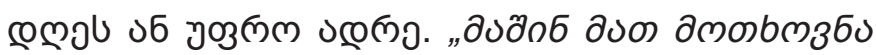

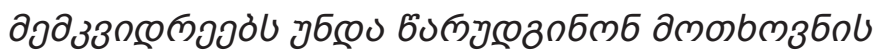

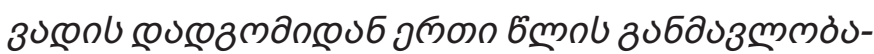

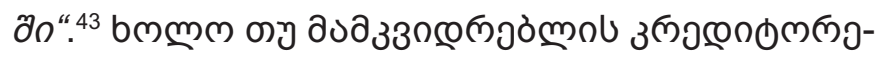

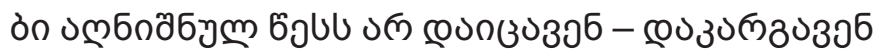
amobmзбol yozmó̀ul. 44

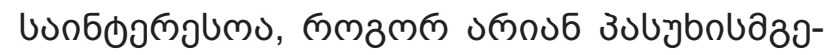

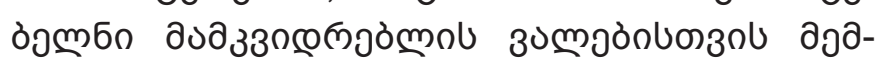

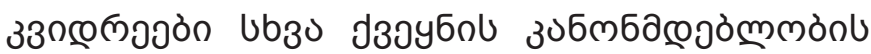

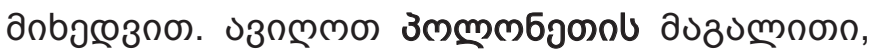

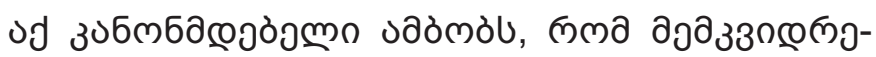

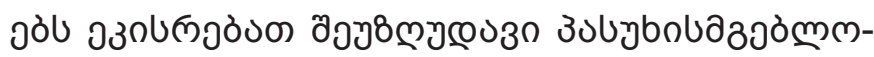

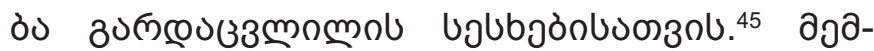

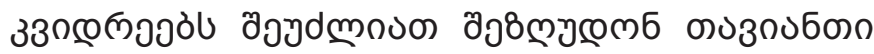

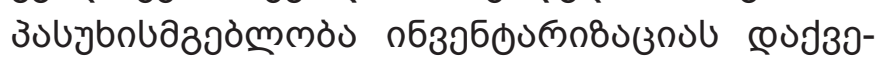

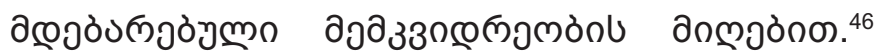

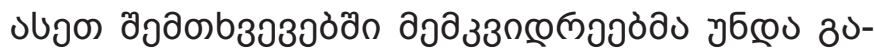

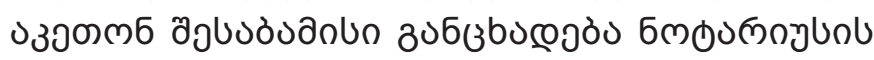

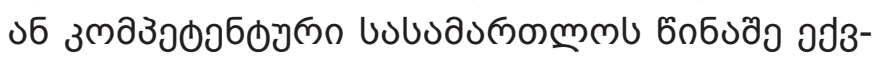

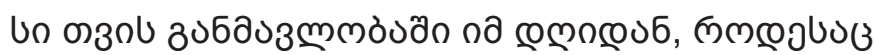

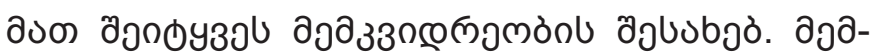

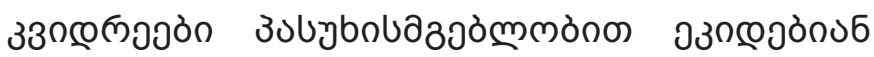

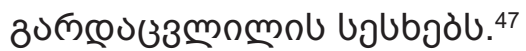

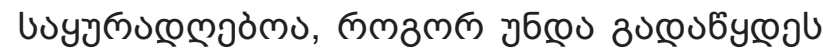

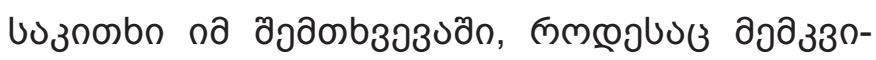

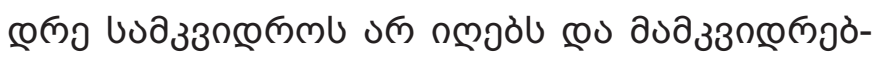

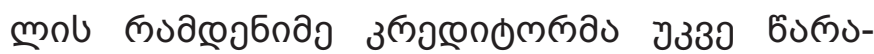

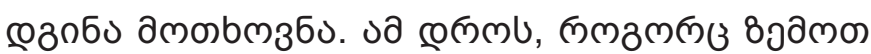

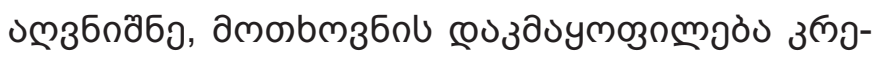

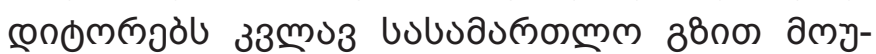

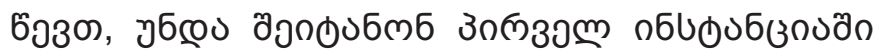

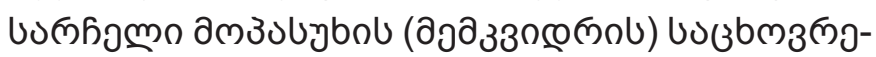

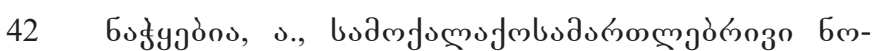

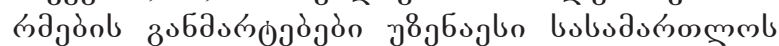

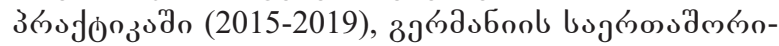

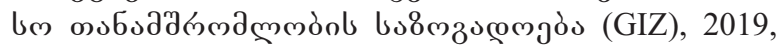

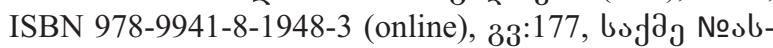

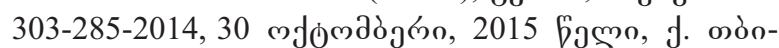
molo.

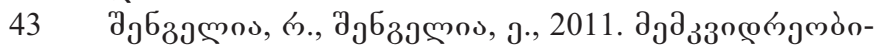

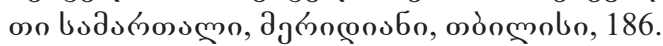

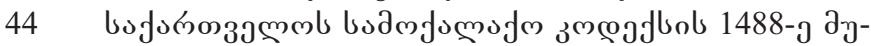
bymo.

45 https://e-justice.europa.eu/content succession-166-pl-en. do\#toc_1 [22.04.2020].

46 คง3ว.

47 oง 39 . 


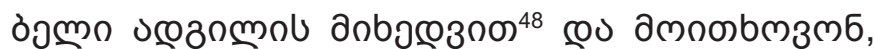

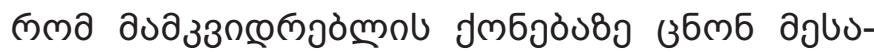

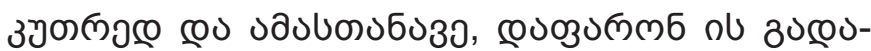

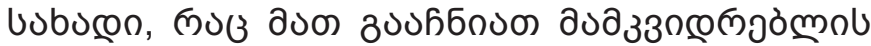

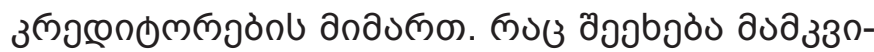

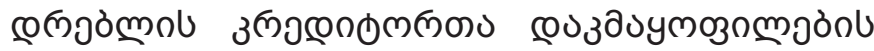

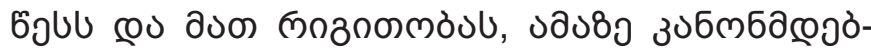

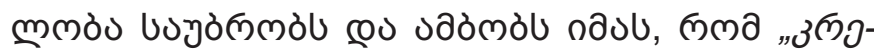

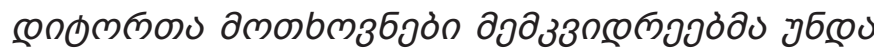

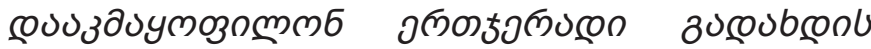

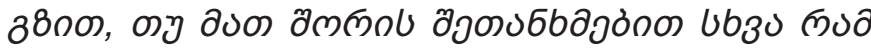

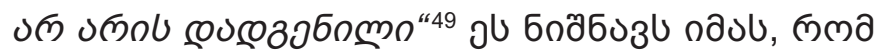

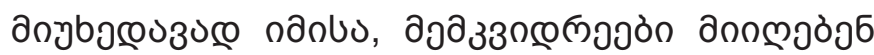

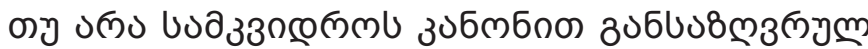

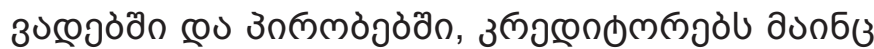

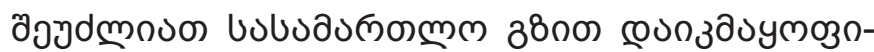

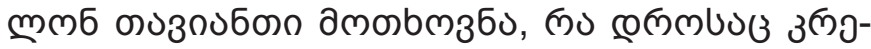

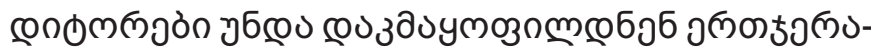

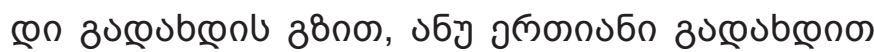

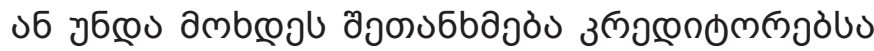

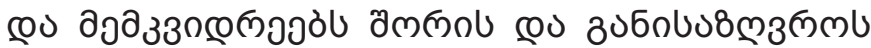

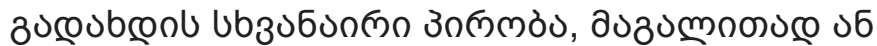

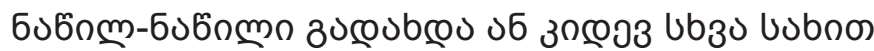

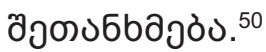

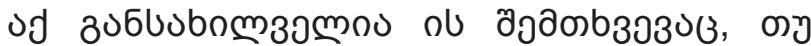

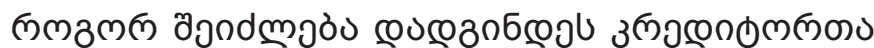

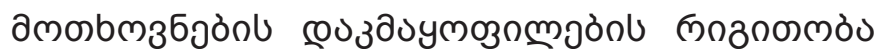

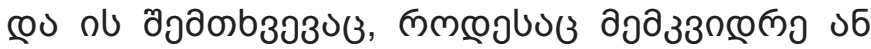

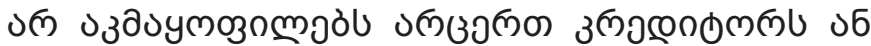

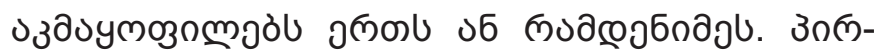

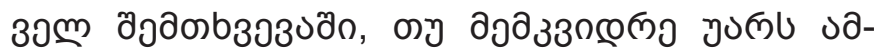

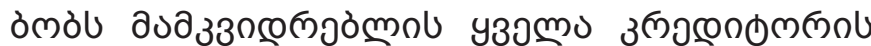

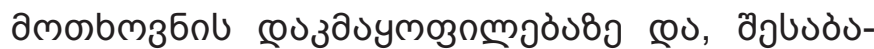

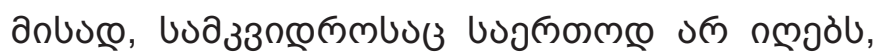

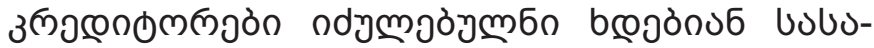

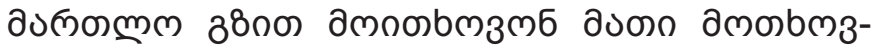

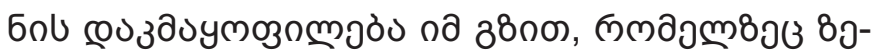

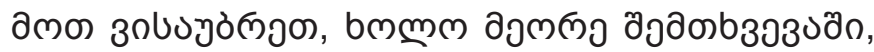

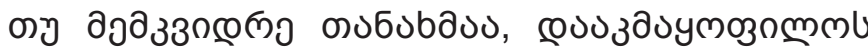

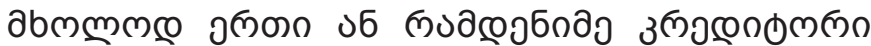

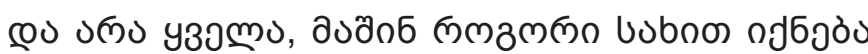

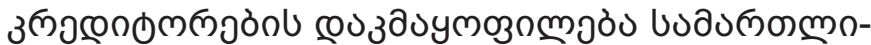

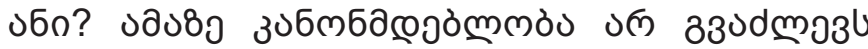

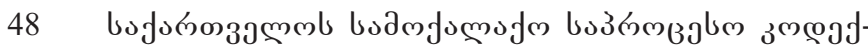
bols $\partial_{9}-15$ aybmo.

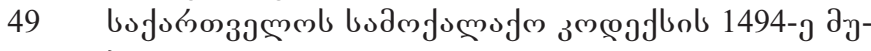
bemo.

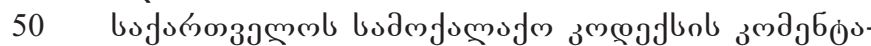

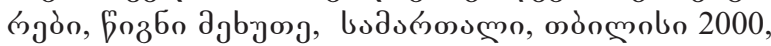
520 .

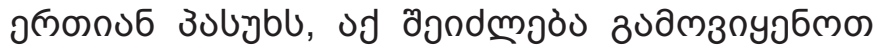

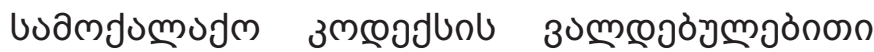

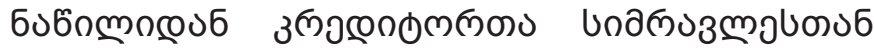

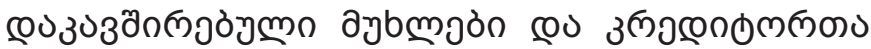

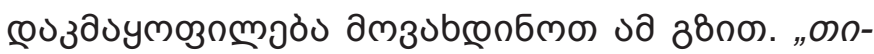

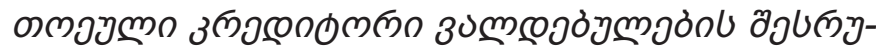

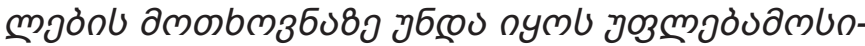

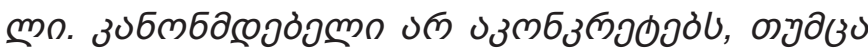

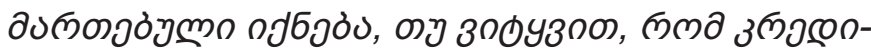

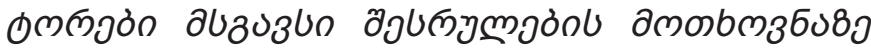

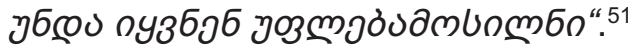

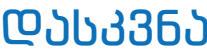

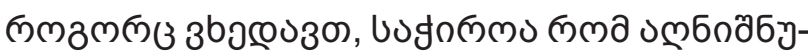

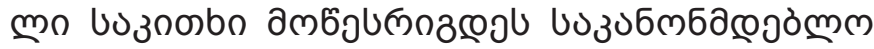

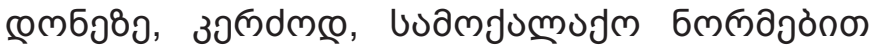

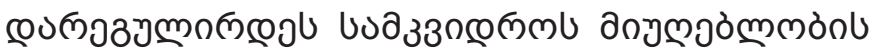

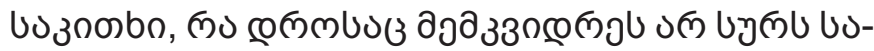

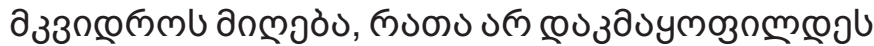

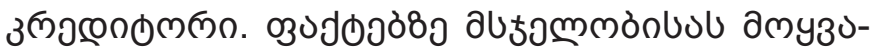

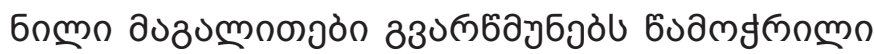

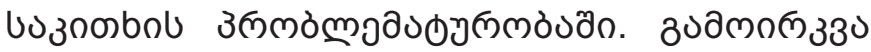

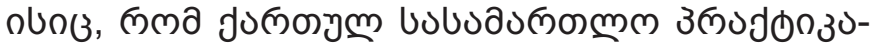

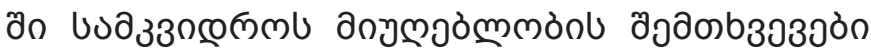

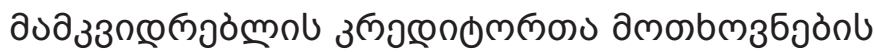

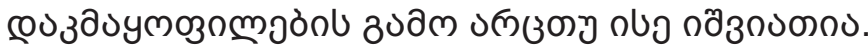

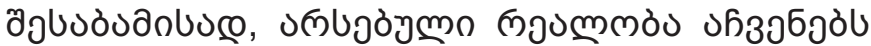

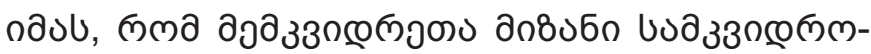

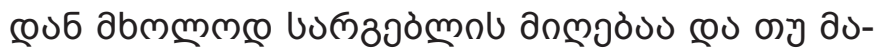

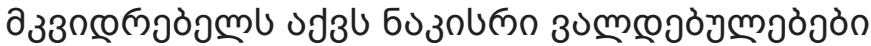

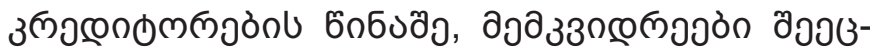

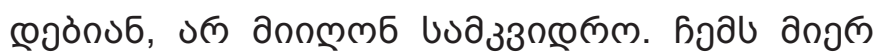

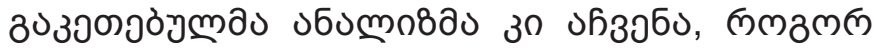

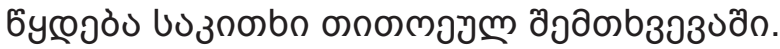

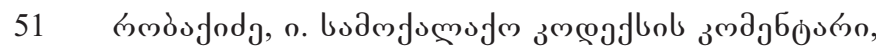
$\partial_{y b \text { mo }} 455$, http://www.gccc.ge/ [20.05.2020]. 


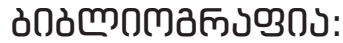

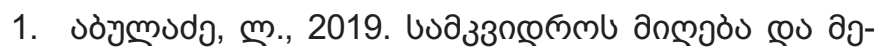

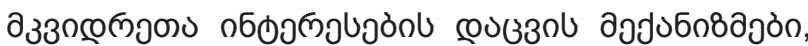

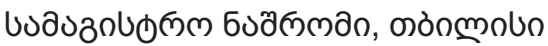

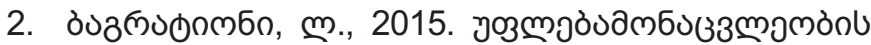

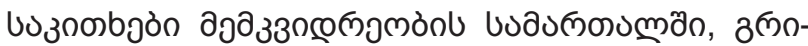

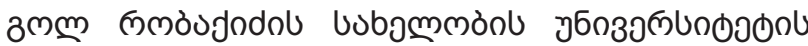

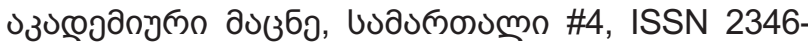
8483 (Online), ònmoun

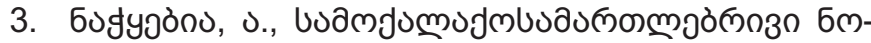

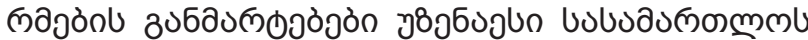

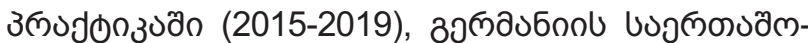

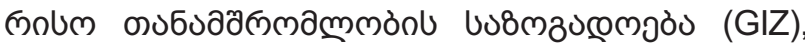
2019, ISBN 978-9941-8-1948-3 (online)

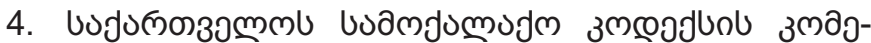

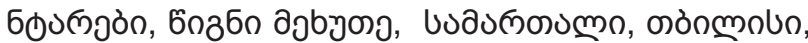
2000

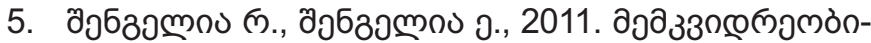

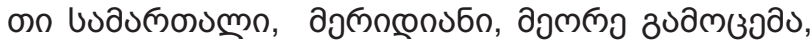
mònmoln

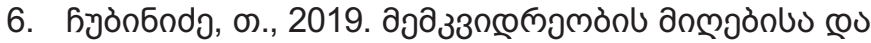

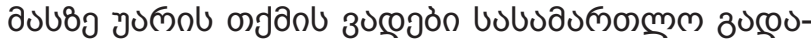

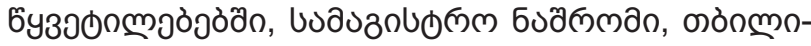
un

7. Adam J. Hirsch, American History of Inheritance Law, OXFORD INTERNATIONAL ENCYCLOPEDIA OF LEGAL HISTORY, Stanley N. Katz ed., 2009

8. Cavanaugh, K.W., 1988. Problems In The Law of Succession: Creditors' Rights, 48 La. L. Review

9. Domingo, R., 2017. The Roman Law of Succession. An Overview, University of Navarra - School of Law

10. Ellul, A., Pagano, M., Panunzi, F., 2010. Inheritance Law and Investment in Family Firms

11. Sousa, L., 2010. The family inheritance process: motivations and patterns of interaction

12. Wormser R. A., Burchard A., 1934. ADMINISTRATION OF GERMAN DECENTENTS' ESTATES

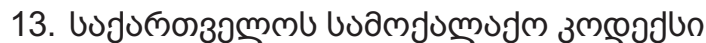

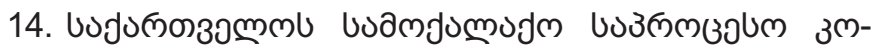
coofun

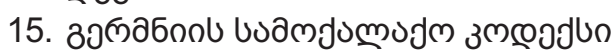

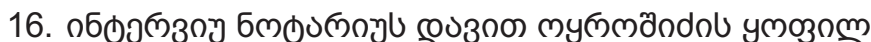

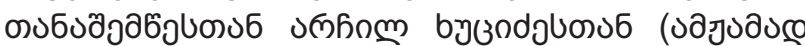

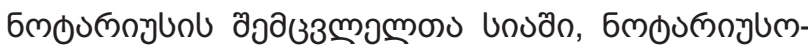

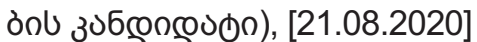

17. https://papers.ssrn.com/sol3/papers.cfm?abstract $\mathrm{id}=982428$ [22.08.2020]

18. https://www.researchgate.net/publication/226734632 The family inheritance process Motivations and patterns of interaction [11.06.2020]

19. https://e-justice.europa.eu/content_succession-166-plen.do\#toc 1 [22.04.2020]

20. http://www.gccc.ge/ [20.05.2020]

\section{BIBLIOGRAPHY:}

1. Abuladze, L., 2019. Inheritance Acceptance and Mechanisms for Protecting the Interests of Heirs, Master Thesis, Tbilisi

2. Bagrationi, L., 2015. Issues of Succession in Inheritance Law, Academic Herald of Grigol Robakidze University, Law \#4, ISSN 2346-8483 (Online), Tbilisi

3. Nachkebia, A., Definitions of Civil Law Norms in the Practice of the Supreme Court (2015-2019), German Society for International Cooperation (GIZ), 2019, ISBN 978-9941-8-1948-3 (online)

4. Comments of the Civil Code of Georgia, Book Five, Law Publishing House, Tbilisi, 2000

5. Shengelia R., Shengelia E., 2011. Inheritance Law, Meridian Publishing House, Second Edition, Tbilisi

6. Chubinidze, T., Terms of Acceptance and Rejection of Inheritance in Court Decisions, Master Thesis, Tbilisi, 2019

7. Adam J. Hirsch, 2009. American History of Inheritance Law, OXFORD INTERNATIONAL ENCYCLOPEDIA OF LEGAL HISTORY, Stanley N. Katz ed

8. Cavanaugh, K.W., 1988, Problems In The Law of Succession: Creditors' Rights, 48 La. L. Review

9. Domingo, R., 2017. The Roman Law of Succession. An Overview, University of Navarra - School of Law

10. Ellul, A., Pagano, M., Panunzi, F., 2010. Inheritance Law and Investment in Family Firms

11. Sousa, L., 2010. The family inheritance process: motivations and patterns of interaction

12. Wormser R. A., Burchard A., 1934. ADMINISTRATION OF GERMAN DECENTENTS' ESTATES

13. Civil Code of Georgia

14. Civil Procedure Code of Georgia

15. Civil Code of Germany

16. Interview with Archil Khutsidze (former on the list of notary substitutes, candidate for notary), assistant of former notary David Okroshidze, [21.08.2020]

17. https://papers.ssrn.com/sol3/papers.cfm?abstract id=982428 [22.08.2020]

18. https://www.researchgate.net/publication/226734632 The family inheritance process Motivations and patterns of interaction [11.06.2020]

19. https://e-justice.europa.eu/content succession-166pl-en.do\#toc 1 [22.04.2020]

20. http://www.gccc.ge/ [20.05.2020] 
NOTES:

1. Bagrationi, L., 2015. Issues of Succession in Inheritance Law, Academic Herald of Grigol Robakidze University, Law \#4, ISSN 2346-8483 (Online), Tbilisi, p. 101

2. Nachkebia, A., Definitions of Civil Law Norms in the Practice of the Supreme Court (2015-2019), German Society for International Cooperation (GIZ), 2019, ISBN 9789941-8-1948-3 (online), p.177

3. Comments of the Civil Code of Georgia, Book Five, Law Publishing House, Tbilisi, 2000, pp. 482, 485, 514, 516, 520

4. Shengelia R., Shengelia E., 2011. Inheritance Law, Meridian Publishing House, Second Edition, Tbilisi, pp.10,158-159, 185, 186

5. Adam, J. Hirsch, 2009. American History of Inheritance Law, OXFORD INTERNATIONAL ENCYCLOPEDIA OF LEGAL HISTORY, Stanley N. Katz ed., p. 235. https://papers.ssrn.com/sol3/papers.cfm?abstract id=982428 [22.08.2020]

6. Cavanaugh, K.W., 1988. Problems In The Law of Succession: Creditors' Rights, 48 La. L. Review, pp. 1100-1101

7. Domingo, R., 2017. The Roman Law of Succession. An Overview, University of Navarra - School of Law, pp. 2-3

8. Ellul, A., Pagano, M., Panunzi, F., 2010. Inheritance Law and Investment in Family Firms, p. 2414

9. Sousa, L., 2010. The family inheritance process: motivations and patterns of interaction, https://www.researchgate.net/publication/226734632_The family inheritance_process_Motivations_and_patterns_of interaction [11.06.2020]

10. Wormser R. A., Burchard A., 1934. ADMINISTRATION OF GERMAN DECENTENTS' ESTATES, pp. 40-41

11. Civil Code of Georgia (articles: 1421, 1435, 1484, 1485, 1343, 1444, 1445, 1492, 1436, 1487, 1488, 1494)

12. Civil Procedure Code of Georgia (article: 15$)$

13. Civil Code of Germany (articles: 1950, 1970, 2346, 2348, 2350, 2351)

14. Interview with Archil Khutsidze (former on the list of notary substitutes, candidate for notary), assistant of former notary David Okroshidze, [21.08.2020]

15. https://e-justice.europa.eu/content_succession-166-pl-en.do\#toc_1 [22.04.2020]

16. http://www.gccc.ge/ [20.05.2020] 